\title{
TMT-Opsins differentially modulate medaka brain function in a context-dependent fashion
}

\section{One Sentence Summary}

Two medakafish non-visual c-type Opsins interact non-additively, impacting the levels of the preprohormone $s s t 1 b$, as well as the voltage-gated sodium channel subunit scn12aa and- at least in part independently of the eyes- the amount of larval day-time rest.

Bruno M. Fontinha ${ }^{1,2}$, Theresa Zekoll ${ }^{1,2}$, Miguel Gallach ${ }^{3+}$, Florian Reithofer ${ }^{1,2^{\wedge}+}$, Alison J. Barker $^{5}$, Maximilian Hofbauer ${ }^{1,2,6}$, Ruth M. Fischer ${ }^{1 \wedge}$, Arndt von Haeseler ${ }^{1,2,3,4}$, Herwig Baier $^{5}$ and Kristin Tessmar-Raible ${ }^{1,2,7^{*}}$

+ equal contribution

${ }^{1}$ Max F. Perutz Laboratories, University of Vienna, Campus Vienna Biocenter, Dr. Bohr-Gasse 9/4, 1030 Vienna, Austria

${ }^{2}$ Research Platform “'Rhythms of Life,' University of Vienna, 1030 Vienna, Austria

3 Center for Integrative Bioinformatics Vienna, Max F. Perutz Laboratories, University of Vienna and Medical University of Vienna, Dr. Bohr-Gasse 9, 1030 Vienna, Austria

4 Bioinformatics and Computational Biology, Faculty of Computer Science, University of Vienna, Vienna, Austria

5 Max Planck Institute of Neurobiology, Martinsried, Germany

${ }^{6}$ loopbio GmbH, Kritzendorf, Austria

${ }^{7}$ FENS-Kavli network of Excellence

present address: 
IMC Fachhochschule Krems, University of Applied Sciences, Krems, Austria

${ }^{\wedge}$ Boehringer Ingelheim RCV GmbH \& Co KG, Vienna, Austria

\title{
Contact Information
}

*kristin.tessmar@mfpl.ac.at

\begin{abstract}
Vertebrate behavior is strongly influenced by light. Photoreceptors, encoded by Opsins, are present inside the vertebrate brain and peripheral tissues. Their non-visual functions are largely enigmatic.

We focus on tmt-opsin $1 b$ and 2, c-Opsins with ancestral-type sequence features, conserved across several vertebrate phyla and with partly similar expression. Their loss-of-function mutations differentially modulate medakafish behavior in a context-dependent manner. Specifically, differences in light conditions have differential effects depending on age and frequency of the light changes, part of which are mediated by TMT-Opsin1b acting outside the eyes, while the pre-pro-hormone $s s t 1 b$ is regulated by daylength via TMT-opsin $1 b$ in an eye-dependent manner. Analyses of tmt-opsin1b;tmt-opsin2 double mutants reveals partial complementation of single mutant behavioral and molecular phenotypes.

Our work starts to disentangle the highly complex interactions of vertebrate non-visual Opsins, suggesting that tmt-opsin-expressing cells together with other Opsins provide detailed light information to the organism for behavioral fine-tuning.
\end{abstract}

Keywords: TMT-opsins, behavior, modulation, transcriptome, teleosts

\section{Introduction}

Organisms are exposed to a large range of light intensities and spectral changes. While humans are well aware of the visual inputs from their environments, the range of intensity and spectral changes that animals, including humans naturally undergo are less consciously experienced. Across the day, light intensity routinely differs by several orders of magnitude depending on the angle of the sun above the horizon and weather conditions (e.g. a $10^{3}$ - 
fold difference occurs between a sunny day and the moments before a thunderstorm, which is a similar difference between a sunny day and the average office illumination)(1).

While vision evolved multiple mechanisms to compensate for these differences, allowing us to see almost equally well across about $10^{6}$ orders of magnitude of light intensity ( 1 , light differences have wider effects on physiology and behavior than solely impacting vision. This has been well documented for vertebrates, including humans (2), covering mood, cognition and fear behavior (3- $)$, but also spanning teleosts (7), frogs ( 8 ) and birds (reviewed in 9).

The past years revealed that Opsins, the proteins mediating light sensation in vertebrates, are present in various cell types inside and outside the vertebrate eyes, including specific brain neurons (10-13). Zebrafish possess 42 opsin genes (14), and additional genomic and transcriptomic data suggest that opsin gene numbers are similarly high in other teleost species (http://www.ensembl.org).

Biochemical analyses, tissue culture assays and electrophysiological recordings on brain tissue suggest that most, if not all, of these Opsins can function as light receptors (13-17). Light has been shown to reach deep brain regions of several mammals and $\operatorname{birds}(10,18)$ (2). Given the typically smaller sizes of fish brains, especially medaka and zebrafish, it is highly conceivable that light will reach cells inside the fish brain. Based on the expression of several Opsins in inter- and motorneurons in larval, as well as the adult zebra- and medaka fish brain we suggested that these Opsins could modulate the information processing of the neurons they are expressed in, depending on ambient light conditions (13).

Studies on young zebrafish larvae indeed implicate non-visual Opsins in specific lightdependent behaviors, particularly in the photomotor response present at 30 hours-postfertilization (hpf) (19), the suppression of spontaneous coiling behavior in larvae younger than 24 hpf (20), as well as part of the dark photokinesis response (in 5-7 days-postfertilization (dpf) old larvae) (12) and the transition between different swimming patterns during extended periods of sudden darkness (in 6-7 dpf old larvae) (21). Yet, non-visual Opsins are not just present during young larval stages, and their later functions remain unclear. Furthermore, another line of evidence suggests that non-visual Opsins are involved in conveying light information for chronobiological functions, such as the circadian clocks 
autonomously present in different fish tissues $(22,23)$. Outside the tropical/ sub-tropical zebrafish, non-visual Opsins have also been suggested to convey photoperiod information, particularly to the gonads (24).

Here we focus on the functional characterization of members of the Encephalopsin/TMTOpsin (ETO) family, which is characterized by a particularly low rate of sequence changes over time and represents ancestral ciliary-type Opsins (13). One of its subfamilies (Encephalopsin) is conserved up to placental mammals $(13,25,26)$. We focused our analyses on medakafish, since these fish show a light-dependent seasonal breeding response as an adaptation to the photoperiod changes naturally occurring in their habitat $(27,28)$. We generated medaka tmt-opsin $1 b$ and tmt-opsin 2 mutants, and analyzed mutant and sibling wildtype fish in multiple behavioral and molecular assays. Our work suggests that these Opsins provide non-redundant environmental light information to the fish, by this modulating a neurohormone and transmitter receptor, as well as behavior. In addition, our work provides several examples how relatively small differences (in age and light intensity) manifests themselves in behavioral changes.

\section{Results}

\section{Ola-tmt-opsin1b mutants exhibit light-dependent altered avoidance responses}

We started our investigation with medaka tmt-opsin $1 b$, by generating several independent mutant alleles using TALEN technology (Fig. 1A). All three mutant alleles are predicted to result in an N-terminally truncated protein, prior to the first transmembrane domain (Supplementary Fig. 1A-D, G) and are thus likely functional null mutants. We confirmed the absence of functional protein by a previously generated antibody against OlaTMTopsin1b (13) (Fig.1B). Given that Ola-TMTopsin1b is expressed in the medaka optic tectum (13) (Fig. 1B), we started our functional analysis with an assay that requires this midbrain structure. Specifically, it has been shown that the responses of frogs and fish to approaching stimuli of different sizes specifically require the tectum and reticular formation(29-33), which both prominently express tmt-opsinlb in medaka (13) (Fig. 1B, 3B,D). We adapted an assay in which the response of individual animals to displayed moving dots of different sizes (mimicking a range of potential prey and predator stimuli) is recorded and analyzed (here referred to as "avoidance assay", Fig. 1C, Supplementary 
Videos 1 and 2). Such type of avoidance assays have been used for toads(29), larval Xenopus(30), mice(34), goldfish(35) and larval zebrafish(33). Medaka larvae of different stages were collected in the morning (ZT2-5) and subjected to the avoidance assay. Hatched medaka larvae are immediately free-swimming and fed. They might best be compared to zebrafish larvae at about $7-8 \mathrm{dpf}$ (constantly raised at $28^{\circ} \mathrm{C}$ ), while later obvious development steps proceed similarly to zebrafish. Based on the responses of the larvae to the moving dots, we calculated an avoidance index (AI) for each dot-size: $\mathrm{AI}=$ (total number of avoidances) - (total number of approaches) divided by (total number of dots presented). Each dot size was presented six times to each fish and care was taken to use different mutant alleles in the same trial (Supplementary Fig. 1H).

After blind response scoring and subsequent genotyping, we observed that tmt-opsin $1 b$ mutant fish exhibited a significantly elevated avoidance response compared to their wildtype siblings, particularly to dot sizes 20 degrees $(P=0.0295$, Unpaired test with Welch's correction; $\mathrm{t}=2.3 ; \mathrm{df}=26.4)$ and 30 degrees $(P=0.0155$, Unpaired $\mathrm{t}$-test; $\mathrm{t}=2.5$; df $=40$ ), decreasing gradually towards bigger dots (Fig. 1D,E). Shuffling dot size order resulted in an overall shift of the response curve, presumably due to habituation of the fish to the larger dots that appeared earlier (compare Fig. 1D,F). Importantly, the elevated response of tmt-opsin $1 b$ mutants vs. wildtype siblings was maintained.

Given that different lab environments resulted in slightly different response curves of the mutant fish (Fig.1D,E) and that TMT-Opsin1b should function as a light receptor in wildtype, we next tested if the observed elevated response in the avoidance assay would be altered by changes of light conditions. The difference of the avoidance responses present between wildtype and tmt-opsin $1 b$ mutants at 100\% light intensity vanishes when the light intensity is reduced (Fig. 1G, $\mathrm{H}, P=0.0318$, Unpaired t-test; $\mathrm{t}=2.2$; $\mathrm{df}=40$, without changing the spectrum, Fig. 1I). Specifically, when we lowered the light intensity of the white background projected by the computer screen to $35 \%$ of the original light intensity, the mutant AI-curve and the wildtype AI-curve (compare Fig. 1E,G,H), are statistically indistinguishable $(P=0.888$, Unpaired t-test; $\mathrm{t}=0.14 ; \mathrm{df}=22)$. We also tested, if the observed changes in the behavioral responses observed between the different light intensity conditions might be due to contrast differences. For this, we analyzed the avoidance index of Cab wildtypes under different contrast conditions (Supplementary Fig. 1I) by testing the 
fish for their responses to dots of increasing luminance (keeping dot size constant at 20 degrees). 35\% lower light levels at the computer screen correspond to a Michelson contrast of 0.93 , a contrast that does not cause any observable behavioral differences compared to full light levels (Supplementary Fig. 1I), making a change of contrast an unlikely explanation for the observed drop in the avoidance index of tmt-opsin $1 b$ mutant fish at 35\% ambient light intensity. These results suggest that tmt-opsin $1 b$ normally mediates constant responses, even under changing light conditions.

\section{Ola-tmt-opsin $1 b$ mutants exhibit altered, partly eye-independent, daytime activity}

\section{levels}

The avoidance assay requires the tectum for proper responses, but it also requires normal functioning eyes. Like probably all Opsins, tmt-opsin $1 b$ is expressed in the eye, specifically in the amacrine layer (13). As the avoidance assay does not allow to discriminate between eye and brain tmt-opsin $1 b$ contributions to the phenotype, we next decided to use an assay that would allow this. Animals typically respond to changes in ambient illumination with rapid changes in movement(36). Changes in motor behavior in response to light in blinded and pinealectomized minnows(7), eels (37) and lamprey tails (38) provided the first evidence for extraretinal, extrapineal "photomotor" behavior in vertebrates. Furthermore, in zebrafish larvae, short periods of sudden darkness result in an increased overall activity, which has been interpreted as 'light-seeking behavior' and termed 'dark photokinesis'(12, 39). Zebrafish larvae lacking the eyes and pineal organ still react to a sudden loss of illumination with an elevated locomotor activity and an undirected light-seeking behavior (12). After a few minutes of continued darkness, zebrafish will subsequently decrease their amount of swimming, resulting in less distance moved during the remaining darkness time $(12,40)$.

We first evaluated the responses of free-swimming tmt-opsin $1 b$ mutant larvae (9-12 dpf) to alternating $1 \mathrm{~min}$ white light and $1 \mathrm{~min}$ dark intervals. Exposure to sudden darkness elicited a "dark photokinesis" response in medaka larvae of both genotypes (Supplementary Fig. 2A, $P<0.0001$, Mann-Whitney test) - further testifying the evolutionary conservation of this innate behavior - but no significant differences between tmt-opsin $1 b$ mutants and wildtype larvae were observed (Supplementary Fig. 2A; $p=$ 0.3516 , Unpaired $\mathrm{t}$ test $\mathrm{t}=0.93 ; \mathrm{df}=174)$, possibly due to a redundancy of photoreceptors 
for this particular assay. Since TMT-Opsins are functional photoreceptors with a maximal sensitivity in the blue light range (about 460nm (13, 41-43), we next modified the assay by using monochromatic blue light, keeping light intensity constant (for spectra see Supplementary Fig. 2B) and by extending the assay time, in order to analyze the expected decline in overall movement upon extended darkness. Mutant vs. wildtype medaka were subjected to $30 \mathrm{~min}$ each of blue light vs. dark intervals (repeated three times), while the movement of the larvae was tracked automatically and evaluated using the Noldus EthoVision $\mathrm{XT}^{\circledR}$ software.

We used fish of two different age groups: 9-12 dpf and 20-22 dpf, representing larvae after the first days post-hatching and juvenile fish with a more "adult-like" morphology, respectively (44).

Both tmt-opsin $1 b$ mutant and wildtype fish changed their swimming distance depending on light / dark condition (Fig. 2A-D), but tmt-opsin1b mutant fish exhibited clear, agedependent differences from the wildtype: while mutant larvae (9-12 dpf) swam less than their wildtype siblings in either light or dark conditions (Fig. 2A,B), their behavior changed gradually during the days post-hatching (Supplementary Fig. 2C-J), resulting in juvenile (20-22 dpf) mutants that swim significantly more than wildtype (Fig. 2C,D).

Analyses of the "dark photokinesis" response rate (first two minutes after light cessation) revealed no difference in the amount of the response between tmt-opsin $1 b$ mutant and wildtype of either age, when the overall lowered or heightened mutant baseline level is accounted for (Supplementary Fig. 2M-N).

We tested the same behavioral paradigm under white light (spectral comparison in Supplementary Fig. 2B). Consistent with a possible redundancy of photoreceptors, we find less pronounced effects at 20-22 dpf (Supplementary Fig. 2K,L). Mutant and wildtype siblings are indistinguishable during the light phases, but they still exhibit significant differences during exposure to sudden darkness (Supplementary Fig. 2K,L).

Given the relatively consistent changes over the entire period of the experiment, we next tested the free-swimming of tmt-opsin $1 b$ mutants across two consecutive days (blue light/dark, 16h:8h). Tmt-opsin1b homozygous mutant larvae and juvenile fish swam significantly more during the $1.5 \mathrm{~h}$ period immediately after the lights went on (Fig. 2E-H). This difference was reduced or disappeared when the light conditions remained stable over 
the rest of the day (Fig. 2E,G). We next tested, if this increase in swimming upon sudden illumination would also occur in fish without eyes, by this testing for a possible functional contribution of tmt-opsin $1 b$ outside the eye (and pineal, as tmt-opsin $1 b$ is not expressed in the pineal). Fish were enucleated and left to recover for 1 week before the trial onset. Importantly, the differences between tmt-opsin $1 b$ and their wildtype counterparts were still observable: while the trend was the same during both days, it reached statistical significance only during the second day (Fig. 2I,J). We thus conclude that tmt-opsin $1 b$, at least in part, functions outside the eyes and modulates the responses of medaka behavior in response to different environmental light changes.

\section{Ola-tmt-opsin $1 b$ and Ola-tmt-opsin 2 mutants exhibit additive and non-additive responses to changes in environmental light}

Teleost, like zebrafish, have been shown to possess more than 40 Opsins, most of which exhibit expression in the brain (14). Tmt-opsin2 is an evolutionarily conserved ETO relative of tmt-opsin $1 b$ with highly similar absorbance characteristics (41) and expression in adjacent, possibly partly overlapping, domains in mid- and hindbrain (13) (Fig. 3B-E). We thus wondered, if mutating tmt-opsin2 would lead to similar phenotypes. Using TALE nucleases, we generated a large deletion in the tmt-opsin2 gene (Fig. 3F), removing the first two transmembrane helices and resulting in a dysfunctional protein (Supplementary Fig. 1E-G). Larval and juvenile tmt-opsin2 mutant and wildtype sibling fish were assessed for their responses during the light / dark phases across two days, as described before. While under these conditions tmt-opsin2-/- mutants do not display significant phenotypes, unexpectedly tmt-opsin 1 b/tmt-opsin 2 double homozygous mutants displayed phenotypes different from the tmt-opsin1b-/- single mutants: while in larval stages adding the tmtopsin 2 mutant to the tmt-opsin $1 b$ mutant resulted in a complementation of the tmt-opsin $1 b$ phenotype during the beginning of the light phases (Fig. 2E,Fand Fig.3G,H), the combination of these mutations lead to additive phenotypes in the same experimental test during juvenile stages (Fig. 2G,H and Fig. 3I,J). This suggests that the different Opsinbased photoreceptors in fish interact in an age-dependent manner to regulate fish behavior. The fact that the tmt-opsin 2 mutation can compensate for tmt-opsin $1 b$ loss during young larval stages also shows that the light information is fed into a complex processing system and the output for behavior is not simply the sum of the input of all possible light receptors. 


\section{Mutations in $t m t-o p \sin 1 b$ and $t m t$-opsin 2 induce transcriptional changes that impact on neuronal information transmission}

We next wondered which molecular changes can be detected in the brain of the fish missing specific photoreceptors. It had previously been shown that differences in photoperiod cause changes on the transcript level in the rat brain, resulting in differences of neurotransmitter abundance(4). We thus reasoned that quantitative RNA sequencing might be a possible strategy to obtain an unbiased insight into the changes that occur due to lack of tmt-opsin $1 b$ or tmt-opsin2 function.

We sampled three separate brain regions (eyes, forebrain and mid-/hindbrain, Fig. 4A) at ZT2-3 under a 16:8 hour white light/dark regime, extracted RNA and sequenced stranded cDNA. It should be noted that for technical reasons part of the mid-/hindbrain sample includes a portion of the posterior hypothalamus, i.e. forebrain. The resulting sequences were mapped to the medaka genome (Ensembl version 96), reads mapping to annotated exons were quantified using edgeR (Bioconductor version 3.9) (45). When comparing tmtopsin $1 b$ and its wildtype siblings, one differentially regulated transcript caught our particular attention, the preprohormone $s s t 1 b$ in the mid-/hindbrain (Fig. 4B), a member of the corticostatin/somatostatin family. We next independently confirmed the quantitative RNAseq results using qPCR (Fig. 4C). In this set of experiments we separated the midbrain from the hindbrain tissue in order to obtain a more differentiated picture of the regulation. Given the partially differential effects of tmt-opsin1b/tmt-opsin2 double mutants on behavior, we next wondered how the presence of both mutations would affect sst $1 b$ regulation. Adding the tmt-opsin 2 mutation compensated the downregulation of $s s t 1 b$ levels present in the tmt-opsin1b single mutant (Fig. 4C). In an analogous approach, we identified the voltage-gated sodium-channel subunit scn12a as significantly regulated by the tmt-opsin 2 mutation (Fig. 4D,E). Again, while clearly visible in the single mutants, the effect was compensated for in the tmt-opsin1b/tmt-opsin2 double mutants (Fig. 4E).

These molecular data further support the notion that the loss-of-function of non-visual Opsins does not lead necessarily to a simple summation effect originating from the single mutants, arguing for non-redundant, complex information processing of the light input, which can modulate the neuronal function on the level of neuropeptides (tmt-opsin $1 b$ regulating sst $1 b$ ) and voltage-gated channels (tmt-opsin2 regulating scn12aa). 


\section{The regulation of $s s t 1 b$ via tmt-opsin $1 b$ is non-cell autonomous}

In order to gain deeper insight on the connection between $s s t 1 b$ expression changes in the mid-hindbrain, tmt-opsin1b and responses to light, we next analyzed the expression of $s s t 1 b$ (Fig. 5). In the midbrain, sst $1 b$ is expressed in several highly specific clusters of cells, none of which overlap with tmt-opsinlb (compare Figs. 5A and 3B). This is particularly obvious for the tectum, in which $s s t 1 b+$ cells are consistently located more dorsally within the stratum periventriculare (SPV) than tmt-opsin1b+ cells, and close to or possibly overlapping with tmt-opsin2+ cells (compare Fig.5A with Fig. 3C). Similarly, in the reticular formation of the hindbrain, likely all of the $s s t 1 b+$ cells are separate from the tmt$o p \sin 1 b+$ cells (Fig. 5B and Fig. 3D). We thus conclude, that the changes of sst $1 b$ transcript levels are rather indirectly mediated by tmt-opsin $1 b+$ cells upstream of $s s t 1 b$-expressing cells.

We next analyzed where the regulation of $s s t 1 b$ transcripts occur. We performed in situ hybridizations on wildtype and tmt-opsin $1 b$ mutant adult fish brains for $s s t 1 b$ with specific attention to treating wildtype and mutant samples identically. We observed a specific reduction of $s s t 1 b+$ transcript levels in the cells of the SPV layer in the optic tectum, the absence of sst $1 b$ staining in the dorsal periventricular hypothalamic zone surrounding the lateral recess and in cells medial to the nucleus glomerulosus in the medial preglomerular nucleus (arrows Fig. 5C). We also observed a reduction of sst $1 b+$ cells in the intermediate reticular formation of the hindbrain (arrows Fig. 5D). These in situ expression analyses suggest that the likely indirect regulation of $s s t 1 b$ transcripts by tmt-opsin $1 b$ occurs in specific cells and thus likely impacts on specific downstream networks.

\section{Photoperiod regulates sst $1 b$ transcript levels via a tmt-opsin $1 b$ dependent mechanism}

We and others have previously provided evidence that TMT-Opsins function as light receptors in tissue culture and upon reconstitution(13, 41-43). The behavioral data presented here further support this view.

Specifically, given that $s s t 1 b$ is regulated by tmt-opsin $1 b$ implies that this transcript should be under light control. We therefore tested, if changes in the illumination regime impact on sst1b transcript levels in a TMT-Opsin1b dependent manner. We chose a 'photoperiod'type light regime based on three reasons: First, any changes in transcription need sufficient time to occur. Second, we wanted to test a light regime that has obvious natural relevance 
to medaka (27), and third, the dynamic somatostatin's expression is regulated in response to exposure to short- and long-day photoperiods in the adult rat (4).

We thus exposed wildtype fish to two different white light regimes (Fig. 6A,B). All fish were initially raised a 16h (light):8h (dark) white light regime (Fig. 6B). One cohort remained exposed to this $16 \mathrm{~h}: 8 \mathrm{~h}$ LD cycle (long day), while the other group was transferred to a $8 \mathrm{~h}: 16 \mathrm{~h}$ LD cycle (short day). After one week, all fish were sacrificed between ZT0 and ZT3, respectively (blue arrowhead Fig. 6A), eyes and brains dissected, RNA prepared and subsequently analyzed by qPCR. A comparison of sst $1 b$ transcript levels at long vs. short day revealed significantly lower transcript level for the short day cohort, mimicking the tmt-opsin 1 b mutation (compare Fig. 6C with Fig. 4B,C). Testing the same photoperiods in the tmt-opsin $1 b$ mutants also revealed that this day length difference depends on functional tmt-opsin $1 b$, as it is abolished in the tmt-opsin $1 b$ mutants (Fig. 6D).

Finally, we addressed if this photoperiod dependent regulation depends on the presence of eyes. We surgically removed the eyes in wildtype adult medaka fish, allowed for 1 week recovery period and subsequently exposed them to the different photoperiods. In contrast to the difference in the behavior that were still observable in eyeless fish (wildtype vs. tmtopsin $1 b$ mutant, Fig. 2I,J), photoperiod differences had no effect on sst $1 b$ transcript levels in the mid-/hindbrain, which were constantly at short day low level (Fig. 6C, E).

This suggests that the photoperiod-dependent regulation of $s s t 1 b$ is primarily mediated by the eyes.

\section{Discussion}

Here we show that the loss-of-function of two non-visual Opsins of the Encephalopsin/TMT-Opsin family leads to distinct light-dependent molecular and behavioral alterations in medaka fish. Focusing mainly on tmt-opsinlb, we show that this Opsin modulates fish swimming behavior during the day partly independently of the eyes (and pineal- as it is not expressed in the pineal (13)). At present, we cannot say which molecular changes are connected to these behavioral changes. Part of the fish behavioral and molecular changes requires the presence of eyes. For these cases we can show that the eye- and tmt-opsin $1 b$ - dependent transcript changes of sst $1 b$ in the mid-/hindbrain are triggered by photoperiod changes. Interestingly, a change of the preprohormone transcript and peptide levels of Somatostatin1 has been reported for long vs. short photoperiods in 
adult rats (4), suggesting a possible evolutionary conservation of this regulatory relationship. Similarly to the changes we observed for sst1b in medaka, SST1 amounts increase when the days are longer. In the case of the rat, this is correlated with a partial decrease of the enzyme tyrosine hydroxylase and the number of dopaminergic neurons, as well as an alteration in stress behavior(4). However, in addition to the changes in the forebrain (posterior hypothalamus), as they were reported for rat, we find $s s t 1 b$ to be significantly regulated in mid- and hindbrain cells. It could be that this also extends to rat and certainly suggests, that brain regions outside the hypothalamus should be analyzed, in order to understand which effects photoperiod differences might exert on mammals. It will then be a question for the future to sort out, which molecular changes are related to which behavioral changes.

One point of note is that the removal of the eyes (with consequential complete degeneration of the optic nerve) abolishes the seasonal light-dependent changes of sst $1 \mathrm{~b}$. This most likely suggests that the response is mediated by the eyes. However, it can at present also not be ruled out that functional eye input is required for the seasonal modulation of $s s t 1 b$ levels to occur, but the light detection itself could happen in cells of the tectum (the main target for retinal ganglion cells, which conveys visual information from the eye to the brain) and hindbrain.

While the similarity to the sst 1 regulation in rats is evolutionarily remarkable, given the widespread occurrence of various photoreceptors in many vertebrate species, e.g (14), it is however likely that 'the one' seasonal light receptor does not exist, but rather multiple light receptors in the eye and different brain regions (and likely outside the brain) contribute to organisms behavioral and physiological adaptations to seasonal changes in light. This is also supported by the combination of behavioral and molecular data presented here.

Given that teleost fish possess many photoreceptors, we here also aimed to obtain a first insight into how these different photoreceptors interact. Unexpectedly for us, both on molecular and behavioral levels, the simultaneous loss of two tmt-opsins could compensate for the loss of each of them individually, suggesting that they act at least in part in opposite directions on the same downstream circuitry. In this respect the expression of tmt-opsin $1 b$, tmt-opsin2 and $s s t 1 b$ in the tectal region is interesting. Tmt-opsin $1 b$ and tmt-opsin2 expression is non-overlapping, both delimiting two distinct cellular populations, within two 
unique layers, while $s s t 1 b$ either overlaps or is directly adjacent to tmt-opsin2 positive cells. This spatial expression raises the possibility that both tmt-opsins and sst $1 b$ might delineate a local neuronal circuit. Future work is needed to further delineate these interactions, which has the additional complexity that at least some of these interactions between different Opsins are also age dependent.

\section{Online Methods}

\section{Medaka fish rearing and ethics statement}

All animal research and husbandry was conducted according to Austrian and European guidelines for animal research (fish maintenance and care approved under: BMWFW66.006/0012-WF/II/3b/2014, experiments approved under: BMWFW-66.006/0003$\mathrm{WF} / \mathrm{V} / 3 \mathrm{~b} / 2016$, which is cross-checked by: Geschäftsstelle der Kommission für Tierversuchsangelegenheiten gemäß $\S 36$ TVG 2012 p. A. Veterinärmedizinische Universität Wien, A-1210 Wien, Veterinärplatz 1, Austria, before being issued by the BMWFW). Medaka fish (Oryzias latipes) strains were kept in a constant recirculating system at approximately $26-28^{\circ} \mathrm{C}$ in a $16 \mathrm{~h}$ light $/ 8 \mathrm{~h}$ dark cycle and bred using standard protocols (46). Collected embryos were kept at $28^{\circ} \mathrm{C}$ until hatching in Embryo Rearing Medium (ERM; 0.1\% (w/v) NaCl, 0.003\% (w/v) KCl, 0.004\% (w/v) $\mathrm{CaCl}_{2} \times 2 \mathrm{H}_{2} \mathrm{O}, 0.016$ $\%$ (w/v) $\mathrm{MgCl}_{2} \times 6 \mathrm{H}_{2} \mathrm{O}, 0.017 \mathrm{mM}$ HEPES and 0.0001\% (w/v) methylene blue). Mutant lines were generated in and outcrossed at least four time to the Cab wildtype background. (tmt-opsin $1 b \Delta+23$ was outcrossed 13 times.) Where available, different mutant alleles were used interchangeably, thereby reducing the probability of effects induced by offtarget mutations.

\section{Design and construction of $t m t$-TALENs}

Transcription activator-like effector nucleases (TALENs) targeting the first exon of medaka TMT-opsin 1b gene (Ensembl gene ENSORLG00000013181) and TMT-opsin2 (Ensembl gene ENSORLG00000012534) were designed using the TALEN targeter prediction tool (https://tale-nt.cac.cornell.edu/; TAL Effector Nucleotide Targeter v2.0) according to the following parameters: equal left and right repeat variable diresidue (RVD) lengths, spacer length of 15-20 bp, NN for G recognition, only T in the upstream base and the presence of a unique restriction site in the spacer region. TAL effector modules were 
assembled and cloned into the array plasmids pFUS using the Golden Gate TALEN and TAL Effector kit (Addgene, Cambridge, USA) according to validated procedure (Cermak et al., 2011). The RVD sequence of the left tmt-opsin 1b-TALEN was HD NN NG NG HD HD HD NI NI HD NN HD NN NI NN HD HD NG binding to the genomic sequence 5' CGTTCCCAACGCGAGCCT-3' and the RVD sequence of the right tmt-opsin 1b-TALEN was NN HD NG HD HD NN HD NG NN HD NN NG HD HD HD HD NN NG binding to the genomic sequence 5'-GCTCCGCTGCGTCCCCGT-3' on the opposite strand featuring a unique BssHII restriction site in the $17 \mathrm{bp}$ spacer region. The RVD sequence of the left tmt-opsin2-TALEN was NN NG NG NG NG HD HD NN NN NG HD NI NN NI HD binding to the genomic sequence 5'-GTTTTCCGGTCAGAC-3' and the RVD sequence of the right tmt-opsin2-TALEN was NI NG HD NN HD NG HD NG NN NN NG NG NN NI binding to the 5'-CATCGCTCTGGTTGA-3' genomic sequence. The final pCS2+ backbone vectors, containing the homodimeric FokI nuclease domains, were used as previously(47). All final TALENs pCS2+ expression vectors were sequence-verified using 5'-TTGGCGTCGGCAAACAGTGG-3' forward and 5' GGCGACGAGGTGGTCGTTGG-3' reverse primers.

\section{Generation of capped TALEN mRNA and microinjection}

Full-length TALEN plasmids were digested with KpnI, gel purified (Gel Extraction kit, Qiagen, Netherlands), and in vitro transcripted (Sp6 mMESSAGE mMACHINE kit, Thermo Fisher Scientifics, USA), followed by a purification using the RNeasy Mini kit (Qiagen). The yield was estimated by NanoDrop (Thermo Fisher Scientific) and diluted to the final concentration for microinjection. Cab strain zygotes were microinjected with a mix containing 5 or $50 \mathrm{ng} / \mu \mathrm{l}$ of each transcribed TALEN mRNA and $0.6 \%$ tetramethylrhodamine (TRITC; Thermo Fisher Scientific) in nuclease-free water.

\section{Genotyping of tmt-opsin $1 b$ and tmt-opsin2-TALEN mutated fish}

Genomic DNA from hatched larvae or caudal fin biopsies were retrieved with lysis buffer (0.1\% SDS; $100 \mathrm{mM}$ Tris/HCl, pH 8.5; 5 mM EDTA; $200 \mathrm{mM} \mathrm{NaCl} ; 0.01 \mathrm{mg} / \mathrm{ml}$ Proteinase $\mathrm{K}$ ) at $60^{\circ} \mathrm{C}$ overnight and $1 \mu \mathrm{l}$ of a 1:20 dilution of the lysate used in a PCR reaction (HotStarTaq, Qiagen). The sequence flanking the tmt-opsin $1 b$ TALEN binding 
sites was amplified using the forward 5'-GGGACTTTCTTTGCGCTTTA-3' and the reverse 5'-CAGGTCAGAGCGGATCTCAT-3' primers. $10 \mu \mathrm{l}$ of the reaction was directly used for restriction digest using 4 units of BssHII enzyme (New England Biolabs, USA) in a $20 \mu \mathrm{l}$ reaction. Genotyping of the tmt-opsin2 locus was made with the forward 5'CGGTGAGCGATGTGACTG-3' and the reverse 5'-GGGAGATCTTTGTCCAGGTG-3' primers. Mutation caused by TALENs was assessed by analyzing the band sizes of the restriction digest product on a $2 \%$ agarose gel. Undigested bands were gel extracted and subcloned into pJet2-1 using the Clone JET PCR Cloning kit (Thermo Fisher Scientific) and sequence-verified.

\section{Immunohistochemiytry}

Dissected brains were fixed in 4\% paraformaldehyde in PBT (PBS with 1\% Triton X-100) for 2 hours, embedded in 3\% agarose and cut into slices of $100 \mu \mathrm{m}$ thickness on a tissue vibratome, followed by 4 hours of blocking in 5\% sheep serum at room temperature. Slices were then incubated for 3 days at $4^{\circ} \mathrm{C}$ with a polyclonal rabbit anti-Ola-TMT-1b antibody (1:250 in 1\% sheep serum). After extensive PBT washes, sections were treated with the secondary antibody goat anti-rabbit Alexa Fluor ${ }^{\circledR} 488$ (Thermo Fischer Scientific) in a 1:500 dilution in 1\% sheep serum supplemented with 1:10000 of 4',6-diamidino-2phenylindole (DAPI), during 2 days at $4{ }^{\circ} \mathrm{C}$. Following PBT washes, slices were mounted in proper mounting medium and pictures were taken on a Zeiss LSM700 confocal scanning microscope.

\section{Avoidance assay}

The behavioral assay was essentially performed as described before(33). A single medaka larva (9 - 12 dpf; unfed) or juvenile fish (20 - 22dpf; fed) was placed in a custom-built acrylic glass chamber (18 x 14 × $305 \mathrm{~mm}$ ) filled with 1x ERM medium (1 cm high), having a transparent bottom and opaque upward walls. Five chambers lying parallel to each other were placed on a horizontal facing computer screen, allowing for the simultaneous recording of 5 free-swimming animals in the same behavioral trial. Stimuli consisted of moving black dots (except when testing for contrast, where different abstract grey values were used) on a white background travelling at a constant speed of $13.5 \mathrm{~mm} / \mathrm{sec}$ in the same direction in every trial. Each behavior trial consisted of 7 blocks of 6 dots each, each block characterized by a unique dot size, presented in a size-ascending or in a pseudo- 
random order. Stimuli were generated on the computer screen using custom-written programs (Ubuntu). Two consecutive blocks were presented with a $19 \mathrm{sec}$ interval, during which no stimulus was displayed. The size of the dots was characterized by its diameter (in pixels) and were calculated as degrees of the larva visual field, as described before(33). A industrial camera (acA1300-30gm GigE; Basler AG, Germany), positioned $40 \mathrm{~cm}$ above the computer screen, allowed the visualization of the fish-dot interactions, recording videos at a frame rate of 33 frames per second for offline analysis. The fish were allowed to accommodate to the chamber for 5 minutes before the beginning of the trial. The experiments were performed during the natural light part of the fish light/dark cycle, between ZT2 and ZT5 and a single animal was used only in one behavior trial to avoid possible habituation (e.g. 48) or learning. Each fish-dot interaction was scored either as an approach or an avoidance when the fish swam toward or away from a moving dot, respectively (33). A neutral interaction was scored when the dot entering the fish visual field cause no change in the initial swimming pattern of the animal or if the fish remained motionless. Approach behaviors consisted of swimming towards the dot, displaying a distinct attraction and predation behavior(48), whereas avoidance behaviors were faster than approaches, characterized by rapid swimming bouts away from the direction of the dot's movement. All video analysis were made prior to genotyping of the fish to shield the identity of the subjects from the observer. An avoidance index [(A.I. = (number avoidances - number approaches) / (number total of dots in a block)] was used as a quantitative behavioral readout. All behavioral assays were made at a constant room temperature of $26^{\circ} \mathrm{C}$, with the room lights switched off and the behavioral setup covered by a black lightimpenetrable cover cloth, this way ensuring that the computer screen would be the only illumination source. The Michelson contrast (49) ((Lmax - Lmin / (Lmax + Lmin)) was used to quantify the relative difference in luminance between the moving dot and the computer screen background. Lmax and Lmin are luminance maximum and minimum, respectively. Medaka are known to have highly acute vision, as demonstrated by previous studies on the optomotor response $(50,51)$, showing excellent visual performance already from hatching (50). 


\section{Photokinesis assay}

Individual larvae (9 to $12 \mathrm{dpf}$; unfed; kept at a $16 \mathrm{~h}: 8 \mathrm{~h}$ light cycle at $28^{\circ} \mathrm{C}$ until hatching), or juvenile medaka fish (20 to $22 \mathrm{dpf}$; fed) were distributed randomly across a 6-well plate containing $10 \mathrm{~mL}$ of 1x ERM medium per well. A behavioral trial consisted of a behavioral paradigm that evaluates the animal swimming activity (i.e. distance moved) during alternating blocks of 30 minutes light and darkness ( 3 hours in total per trial)(52), at a constant temperature of $27^{\circ} \mathrm{C}$. An initial acclimation phase of $5 \mathrm{~min}$ to darkness was used before the start of the trial. When assessing the swimming responses in a more naturalisticlike assay, a photoperiod of $16 \mathrm{~h}$ light / $8 \mathrm{~h}$ dark was used during 2 consecutive days, at a constant temperature of $27^{\circ} \mathrm{C}$. Behavioral assessment was made between ZT3 and ZT12. DanioVision $^{\mathrm{TM}}$ (Noldus, The Netherlands) hardware was used to track the distance moved by the animals in each trial. Larval motion was tracked at 60 frames/s over the trial. In an alternate experiment, $1 \mathrm{~min}$ pulses of white light and darkness were presented to the larvae, during a total trial duration of 7 minutes (refers to Supplementary Fig. 2A). Video data was posteriorly analyzed offline by the tracking software EthoVision $\mathrm{XT}^{\circledR}$ (Noldus) to calculate the average distance moved by the animal every $10 \mathrm{sec}$ of the trial. When comparing the dark photokinesis between tmt-opsin1b mutant and wildtype siblings (refers to Supplementary Fig. 2M,N), we normalized the different baseline levels by taking the average distance moved during the 5 minutes preceding darkness along the trial as a normalization factor. Each animal was used only once per trial, thus avoiding possible habituation biases. The ILT950 spectrometer (International Light Technologies Inc., USA) was used to measure the spectra and intensity of the different light sources. A lightimpenetrable cloth was placed over the behavior setup, thus reassuring a lack of external light contamination.

\section{Diel swimming test}

Care was taken to start each trial at the precise light-to-dark transition, thus minimizing any deleterious effect that the shift to a new environment might impose on the natural behavior of the fish. Since scheduled food availability has an impact on the locomotor activity of fish (53), animals were kept unfed during the two consecutive days of the experiment, thus ensuring that the change in ambient light was the central feature impacting 
on swimming responses. The temperature was kept constant at $27^{\circ} \mathrm{C}$. The setup, tracking and off-line analysis was made as previously described for the "photokinesis assays", with the exception that the activity of the animals was averaged every 1 minute.

\section{Enucleations}

Enucleations were performed on $13 \mathrm{dpf}$ wildtype and tmt-opsin $1 b$ mutant juvenile fish upon anaesthetization with $0.03 \%$ tricaine (MS-222) (Sigma, St. Louis, MO). After the surgery, fish were allowed to recover overnight in fresh rearing fish water at $26^{\circ} \mathrm{C}$. After 1 week post-surgery, the fish were used for behavior testing. Handling control fish were anaesthetized for the same duration and placed afterwards in fresh fish water overnight.

\section{RNA extraction and sequencing}

For RNA-seq experimentation, the eyes, forebrain, midbrain and hindbrain (anatomical boundaries according to (54) were dissected from age-matched wildtype and mutant tmtopsin $1 b$ and tmt-opsin 2 adult (>2 months old) medaka sibling fish. One metal bead (Peqlab Biotechnologie GmbH, Germany) and $350 \mu \mathrm{RLT}$ buffer (Qiagen) including 1\% $\beta$ mercaptoethanol was added to the frozen tissue parts, followed by homogenization for 3 minutes at $30 \mathrm{~Hz}$ using the Qiagen tissue lyser. Total RNA was extracted using the RNeasy Mini kit (Qiagen) according to manufacturer's protocol. Three independent biological replicates, each made of 3 individual fish (total of 9 fish per genotype) were used. For tmt$o p \sin 1 b, 1$ fish per independent mutation was used. The quality of total RNA was checked using the Agilent RNA 6000 Nano kit (Agilent, USA) and then enriched for poly(A)+ RNA using the Dynabeads ${ }^{\circledR}$ mRNA Purification Kit (Thermo Fisher Scientific). We used the SuperScript ${ }^{\circledR}$ VILO cDNA Synthesis Kit (Thermo Fisher Scientific) to generate strandspecific cDNA that was further sequenced on the Illumina Hiseq2500 platform by the VBCF NGS Unit (www.vbcf.ac.at) as 100 base single-end reads, resulting on a 15-61 million reads, on average, per biological replicate.

\section{Differential gene expression}

Sequences from each sample were mapped against the assembled chromosomes of the medaka genome (Ensembl version 96) using the read mapper NextGenMap (55). After filtering for duplicates and low quality base, the stand-alone featureCounts tool (56) was used to count mapped reads per each transcript in each sample. The Bioconductor package 
edgeR (version 3.9) was used to analyze read count data and to identify differential expression levels (Benjamini-Hochberg method). We classified genes as being differentially expressed between genotypes when the differences in expression level between wildtype and homozygous mutant fish were significant at a false discovery rate of $5 \%$.

\section{Quantitative PCRs}

One metal bead (Peqlab Biotechnologie) and $700 \mu 1$ TRI Reagent ${ }^{\circledR}$ (Sigma-Aldrich, St. Louis, USA) were added to the previously dissected and frozen eyes, forebrain, midbrain and hindbrain, followed by homogenization for 2 minutes at $30 \mathrm{~Hz}$ using the Qiagen tissue lyser. Total RNA was extracted from the different brain parts and eyes using the DirectZol $^{\mathrm{TM}}$ RNA MiniPrep (Zymo Research, USA) according to the manufacturer's protocol. $80 \mathrm{ng}$ of total RNA was initially used to be transcribed into cDNA using the QuantiTect Reverse Transcription kit (Qiagen) with random hexamer primers. Each cDNA was further analyzed in duplicate in a $25 \mathrm{ul}$ volume using a SYBR green-containing mastermix in the Applied Biosystems (Thermo Fisher Scientific) mastercycler. Intron spanning qPCR primers were designed with the universal probe library software from Roche. Expression of each gene was normalized to beta-actin ) transcript levels and fold changes were calculated.

Table 1: Forward and reverse primer sequences used for qPCR

\begin{tabular}{|c|c|c|c|}
\hline Gene & Forward primer $\left(5^{\prime}-->3^{\prime}\right)$ & Reverse primer $\left(5^{\prime}-->3^{\prime}\right)$ & Ensembl Gene ID \\
\hline sst $1 b$ & ggcttcctgtggaggaca & cagacaccagcttaaggatca & $\begin{array}{l}\text { ENSORLG0000002773 } \\
6\end{array}$ \\
\hline beta-actin & gtgctgtctttccetccatc & tagctgtctttctggeccat & $\begin{array}{l}\text { ENSORLG0000001367 } \\
6\end{array}$ \\
\hline $\operatorname{scn} 12 a a$ & gcagatgacctgtcggaact & ctgacagtgcctcagacagaa & $\begin{array}{l}\text { ENSORLG0000001167 } \\
7\end{array}$ \\
\hline
\end{tabular}




\section{In situ hybridization (ISH) on adult brain sections}

The generation of antisense digoxigenin (DIG)-labeled RNA probes and the ISH staining procedure on adult brain sections were performed as previously (13). Briefly, adult fish (> 2 months-old) were anesthetized in fish water containing $0.2 \%$ tricaine, decapitated, and the brain dissected, subsequently fixed in ice-cold 4\% PFA overnight. After rehydration in 1X PTW (PBS + 0.1\% Tween-20), brains were digested with fresh $10 \mu \mathrm{g} / \mathrm{ml}$ Proteinase K (Merck, USA) for 35 minutes, followed by a 4 hours prehybridization step at $65^{\circ} \mathrm{C}$ with Hyb + solution (50\% Formamide, 5x SSC ( $\mathrm{pH}=6.0), 0.1 \%$ Tween-20, $0.5 \mathrm{mg} / \mathrm{ml}$ torula (yeast) RNA, $50 \mu \mathrm{g} / \mathrm{ml}$ Heparin). Incubation with the specific DIG-RNA probe was made overnight at $65^{\circ} \mathrm{C}$. Coronal whole-brain slices (100 $\mu \mathrm{m}$ thickness) were made using a vibrating blade microtome (Leica VT1000S, Leica Biosystems, Germany), blocked for > 1 hour in 10\% sheep serum / 1x PTW, followed by incubation with 1:2000 dilution of antiDIG-alkaline phosphatase-coupled antibody (Roche, Switzerland) in 10\% sheep serum overnight. Detection of DIG-probes was made in staining buffer (in 10\% polyvinyl alcohol) supplemented with nitro-blue tetrazolium (NBT) and 5-bromo-4-chloro-3'indolyphosphate (BCIP) (Roche).

\section{Statistics}

The D'Agostino \& Pearson normality test was used to asses data distribution. The F-test for comparison of variances for normally distributed data was used to assess the variance of the standard deviation. When comparing two normally distributed groups of data with the same standard deviation the unpaired $t$ test was used. For comparing two groups of normally distributed data from with different standard deviations, the unpaired t test with Welch correction was used. The Mann-Whitney (M-W) test was used when comparing two non-normal distributed data sets. The software used was Prism version 8.

Data is presented as mean \pm s.e.m. For all statistical tests used, a two-tailed $P$ value was chosen: * $P \leq 0.05$; ** $P \leq 0.01$; *** $P \leq 0.001$; **** $P \leq 0.0001$ 


\section{Author contributions}

B.M.F. performed the experiments and analyzed the data. T.Z. performed part of the behavior and qPCR experiments. F.R. performed part of the qPCR experiments. M.G. and A.v.H mapped the transcriptome reads and performed the differential expression seq analyses. A.J.B. and H.B contributed equipment and analysis expertise for the avoidance assay. M.H. constructed the dot assay setup and contributed with software programming. R.M.F generated the tmt-opsin $1 b$ and tmt-opsin 2 mutants. B.M.F and K.T-R designed the study, interpreted the data and wrote the manuscript.

\section{Acknowledgements}

We thank Andrij Belokurov and Margaryta Borisova for fish care, Andrew Straw and the members of the Tessmar-Raible, Raible, von Haeseler and Baier labs for helpful discussions, Mario Wullimann for advice on medaka neuroanatomy, Mirta Resetar for preparation of fish brains and RNA for DEseq analyses and Sven Schenk for sharing the mRNA sequencing protocol. Marin Čagelj contributed with the illustrations on Figures $1 \mathrm{C}$, 3A, 4A. This work was supported by the research platform "Rhythms of Life" of the University of Vienna to K.T-R and A.v.H, an FWF (http://www.fwf.ac.at/) FWF START award (\#AY0041321) and research project grant (\#P28970), and funding from the European Research Council under the European Community's Seventh Framework Programme (FP7/2007-2013)/ERC Grant Agreement 337011 to K.T-R. A.J.B. and H.B. acknowledge funding by SFB 870 „Assembly and Function of Neural Circuits" and the Max Planck Society. R.M.F. was supported by a PhD fellowship of the Boehringer Ingelheim Foundation. The funders had no role in study design, data collection and analysis, decision to publish, or preparation of the manuscript.

\section{Competing Financial Interests}

The authors declare no competing financial interests. 


\section{References}

1. T. Posch, A. Freyhoff, T. Uhlmann, Das Ende der Nacht: Die globale Lichtverschmutzung und ihre Folgen. (Wiley VCH, Weinheim, ed. 1st edition, 2010), pp. 154.

2. D. M. Hunt, M. W. Hankins, S. P. Collin, N. J. Marshall, Evolution of visual and non-visual pigments. (Springer, New York, 2014), pp. 276.

3. D. C. Fernandez et al., Light Affects Mood and Learning through Distinct RetinaBrain Pathways. Cell 175, 71-84 e18 (2018).

4. D. Dulcis, P. Jamshidi, S. Leutgeb, N. C. Spitzer, Neurotransmitter switching in the adult brain regulates behavior. Science 340, 449-453 (2013).

5. G. W. Lambert, C. Reid, D. M. Kaye, G. L. Jennings, M. D. Esler, Effect of sunlight and season on serotonin turnover in the brain. Lancet 360, 1840-1842 (2002).

6. A. R. Webb, Considerations for lighting in the built environment: Non-visual effects of light. Energy and Buildings 38, 721-727 (2006).

7. K. von Frisch, Beiträge zur Physiologie der Pigmentzellen in der Fischhaut. Pflüg. Arch. ges. Physiol. 138, 319-387 (1911).

8. S. P. Currie, G. H. Doherty, K. T. Sillar, Deep-brain photoreception links luminance detection to motor output in Xenopus frog tadpoles. Proc. Natl. Acad. Sci. U. S. A. 113, 6053-6058 (2016).

9. A. Dawson, V. M. King, G. E. Bentley, G. F. Ball, Photoperiodic control of seasonality in birds. J. Biol. Rhythms 16, 365-380 (2001).

10. R. G. Foster, B. K. Follett, J. N. Lythgoe, Rhodopsin-like sensitivity of extraretinal photoreceptors mediating the photoperiodic response in quail. Nature 313, 50-52 (1985).

11. S. Halford et al., VA opsin-based photoreceptors in the hypothalamus of birds. Curr. Biol. 19, 1396-1402 (2009).

12. A. M. Fernandes et al., Deep brain photoreceptors control light-seeking behavior in zebrafish larvae. Curr. Biol. 22, 2042-2047 (2012).

13. R. M. Fischer et al., Co-expression of VAL- and TMT-opsins uncovers ancient photosensory interneurons and motorneurons in the vertebrate brain. PLoS Biol. 11, e1001585 (2013).

14. W. I. L. Davies et al., An extended family of novel vertebrate photopigments is widely expressed and displays a diversity of function. Genome Res. 25, 16661679 (2015).

15. A. Jenkins et al., VA opsin, melanopsin, and an inherent light response within retinal interneurons. Current Biology : $C B$ 13, 1269-1278 (2003).

16. W. I. Davies et al., Functional diversity of melanopsins and their global expression in the teleost retina. Cell. Mol. Life Sci. 68, 4115-4132 (2011).

17. Y. Nakane, T. Shimmura, H. Abe, T. Yoshimura, Intrinsic photosensitivity of a deep brain photoreceptor. Curr. Biol. 24, R596-597 (2014).

18. E. E. V. Brunt, M. D. Shepherd, J. R. Wall, W. F. Ganong, M. T. Clegg, Penetration of light into the brain of mammals. Ann. N. Y. Acad. Sci. 117, 217-224 (1964).

19. D. Kokel et al., Identification of nonvisual photomotor response cells in the vertebrate hindbrain. The Journal of Neuroscience 33, 3834-3843 (2013). 
20. D. Friedmann, A. Hoagland, S. Berlin, E. Y. Isacoff, A spinal opsin controls early neural activity and drives a behavioral light response. Curr. Biol. 25, 69-74 (2015).

21. E. J. Horstick, Y. Bayleyen, J. L. Sinclair, H. A. Burgess, Search strategy is regulated by somatostatin signaling and deep brain photoreceptors in zebrafish. BMC Biol. 15, (2017).

22. N. Cavallari et al., A blind circadian clock in cavefish reveals that opsins mediate peripheral clock photoreception. PLoS Biol. 9, e1001142 (2011).

23. H. A. Moore, D. Whitmore, Circadian Rhythmicity and Light Sensitivity of the Zebrafish Brain. PLoS ONE 9, (2014).

24. Y. Nakane et al., The saccus vasculosus of fish is a sensor of seasonal changes in day length. Nat. Commun. 4, (2013).

25. S. Blackshaw, S. H. Snyder, Encephalopsin: a novel mammalian extraretinal opsin discretely localized in the brain. J. Neurosci. 19, 3681-3690. (1999).

26. J. Nissila et al., Encephalopsin (OPN3) protein abundance in the adult mouse brain. J. Comp. Physiol. A Neuroethol. Sens. Neural. Behav. Physiol. 198, 833839 (2012).

27. M. Awaji, I. Hanyu, Seasonal Changes in Ovarian Response to Photoperiods in Orange-Red Type Medaka : Reproductive Biology. Zoological Science 6, 943950 (1989).

28. C. S. Koger, S. J. Teh, D. E. Hinton, Variations of light and temperature regimes and resulting effects on reproductive parameters in medaka (Oryzias latipes). Biol. Reprod. 61, 1287-1293 (1999).

29. J. P. Ewert, Neural Mechanisms of Prey-Catching and Avoidance Behavior in Toad (Bufo-Bufo L). Brain Behavior and Evolution 3, 36-\& (1970).

30. W. Dong et al., Visual Avoidance in Xenopus Tadpoles Is Correlated With the Maturation of Visual Responses in the Optic Tectum. J. Neurophysiol. 101, 803815 (2009).

31. A. S. Khakhalin, D. Koren, J. Gu, H. Xu, C. D. Aizenman, Excitation and inhibition in recurrent networks mediate collision avoidance in Xenopus tadpoles. Eur. J. Neurosci. 40, 2948-2962 (2014).

32. J. L. Semmelhack et al., A dedicated visual pathway for prey detection in larval zebrafish. Elife 3, (2014).

33. A. J. Barker, H. Baier, Sensorimotor Decision Making in the Zebrafish Tectum. Curr. Biol. 25, 2804-2814 (2015).

34. M. Yilmaz, M. Meister, Rapid Innate Defensive Responses of Mice to Looming Visual Stimuli. Curr. Biol. 23, 2011-2015 (2013).

35. T. Preuss, P. E. Osei-Bonsu, S. A. Weiss, C. Wang, D. S. Faber, Neural representation of object approach in a decision-making motor circuit. J. Neurosci. 26, 3454-3464 (2006).

36. G. S. Fraenkel, D. L. Gunn, The Orientation of Animals, Kineses, Taxes and Compass Reactions. . (Dover Publications Inc., New York, 1961).

37. T. Van Veen, H. Hartwig, K. Müller, Light-dependent motor activity and photonegative behavior in the eel (Anguilla anguilla L.). Journal of comparative physiology 111, 209-219 (1976). 
38. J. Young, The photoreceptors of lampreys: I. Light-sensitive fibres in the lateral line nerves. J. Exp. Biol. 12, 229-238 (1935).

39. H. A. Burgess, M. Granato, Modulation of locomotor activity in larval zebrafish during light adaptation. J. Exp. Biol. 210, 2526-2539 (2007).

40. D. A. Prober, J. Rihel, A. A. Onah, R. J. Sung, A. F. Schier, Hypocretin/orexin overexpression induces an insomnia-like phenotype in zebrafish. J. Neurosci. 26, 13400-13410 (2006).

41. K. Sakai, T. Yamashita, Y. Imamoto, Y. Shichida, Diversity of Active States in TMT Opsins. PLoS ONE 10, e0141238 (2015).

42. T. Sugihara, T. Nagata, B. Mason, M. Koyanagi, A. Terakita, Absorption Characteristics of Vertebrate Non-Visual Opsin, Opn3. PLoS ONE 11, e0161215 (2016).

43. M. Kato et al., Two Opsin 3-Related Proteins in the Chicken Retina and Brain: A TMT-Type Opsin 3 Is a Blue-Light Sensor in Retinal Horizontal Cells, Hypothalamus, and Cerebellum. PLoS ONE 11, e0163925 (2016).

44. M. Kinoshita, K. Murata, K. Naruse, M. Tanaka, in Medaka: Biology, Management, and Experimental Protocols, M. Kinoshita, K. Murata, K. Naruse, M. Tanaka, Eds. (Wiley-Blackwell, 2009), pp. 165-276.

45. M. D. Robinson, D. J. McCarthy, G. K. Smyth, edgeR: a Bioconductor package for differential expression analysis of digital gene expression data. Bioinformatics 26, 139-140 (2010).

46. M. Kinoshita, K. Murata, K. Naruse, M. Tanaka, Medaka: Biology, Management, and Experimental Protocols. (Wiley-Blackwell, 2009).

47. S. Bannister et al., TALE Nucleases mediate efficient, heritable genome modifications in the marine annelid Platynereis dumerilii. Genetics 197, 19-31 (2014).

48. W. Matsunaga, E. Watanabe, Habituation of medaka (Oryzias latipes) demonstrated by open-field testing. Behav. Processes 85, 142-150 (2010).

49. A. A. Michelson, Studies in optics. (Chicago, Ill. : The University of Chicago Press, 1927).

50. J. C. Beck, E. Gilland, D. W. Tank, R. Baker, Quantifying the ontogeny of optokinetic and vestibuloocular behaviors in zebrafish, medaka, and goldfish. $J$. Neurophysiol. 92, 3546-3561 (2004).

51. P. S. M. Carvalho, D. B. Noltie, D. E. Tillitt, Ontogenetic improvement of visual function in the medaka Oryzias latipes based on an optomotor testing system for larval and adult fish. Anim. Behav. 64, 1-10 (2002).

52. D. Kokel et al., Rapid behavior-based identification of neuroactive small molecules in the zebrafish. Nat. Chem. Biol. 6, 231-237 (2010).

53. J. F. Lopez-Olmeda, E. V. Tartaglione, H. O. de la Iglesia, F. J. SanchezVazquez, Feeding entrainment of food-anticipatory activity and per1 expression in the brain and liver of zebrafish under different lighting and feeding conditions. Chronobiol. Int. 27, 1380-1400 (2010).

54. R. Anken, F. Bourrat, Brain atlas of the Medakafish Oryzias latipes. (Institut national de la recherche agronomique, Paris, 1998). 
55. F. J. Sedlazeck, P. Rescheneder, A. von Haeseler, NextGenMap: fast and accurate read mapping in highly polymorphic genomes. Bioinformatics 29, 2790-2791 (2013).

56. Y. Liao, G. K. Smyth, W. Shi, featureCounts: an efficient general purpose program for assigning sequence reads to genomic features. Bioinformatics $\mathbf{3 0}$, 923-930 (2014). 


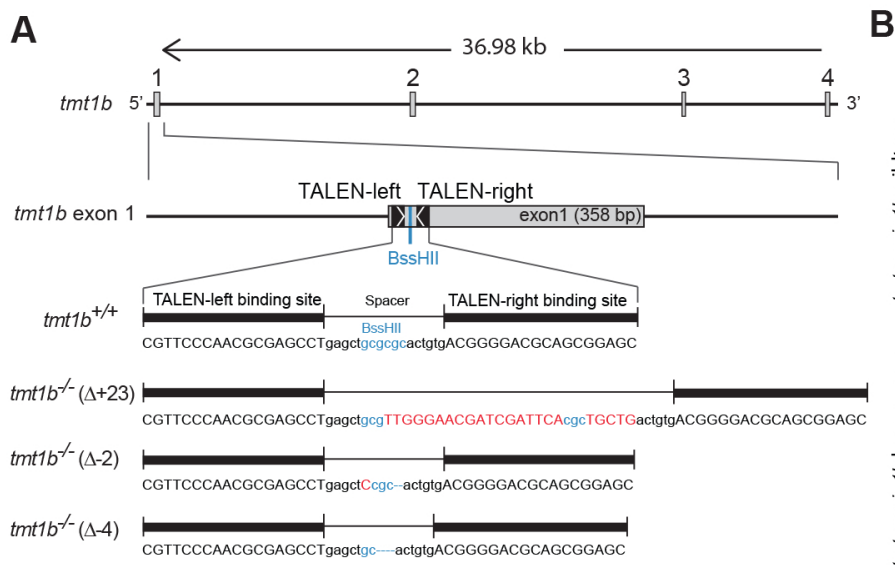

C

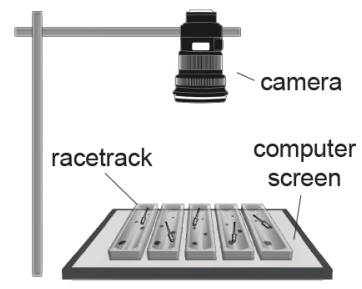

G

$35 \%$ light intensity

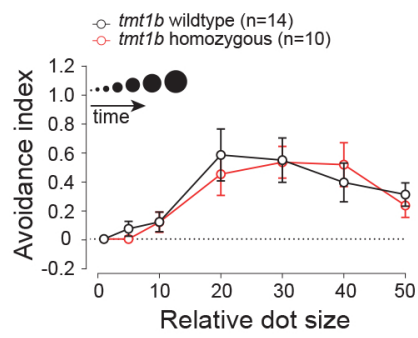

D

$100 \%$ light intensity

- tmt1b wildtype ( $\mathrm{n}=31$ )

- tmt1b homozygous $(n=21)$

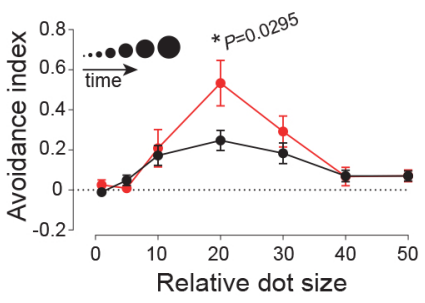

H

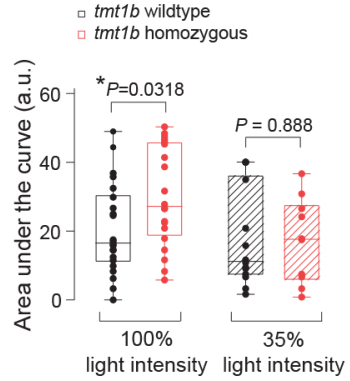

E
B

$100 \%$ light intensity

$\mathbf{F}$

- tmt1 $1 b$ wildtype $(n=22)$

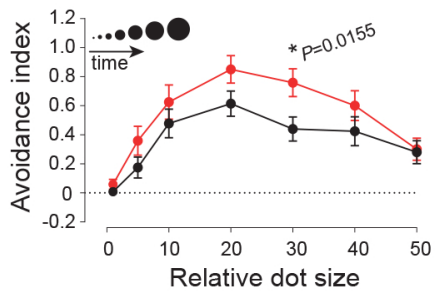

$100 \%$ light intensity

- $t m t 1 b$ wildtype $(n=8$

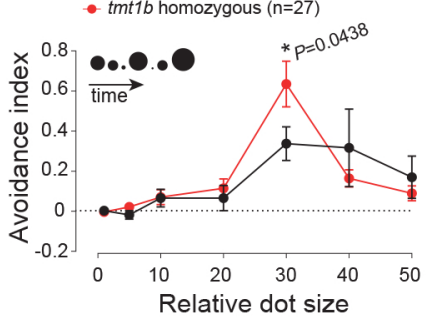

I

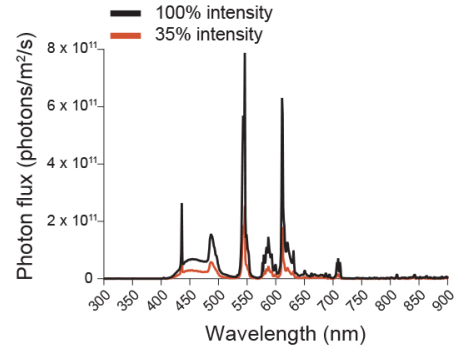


A

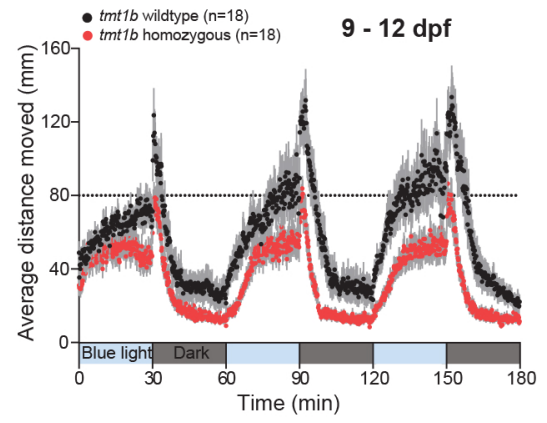

E

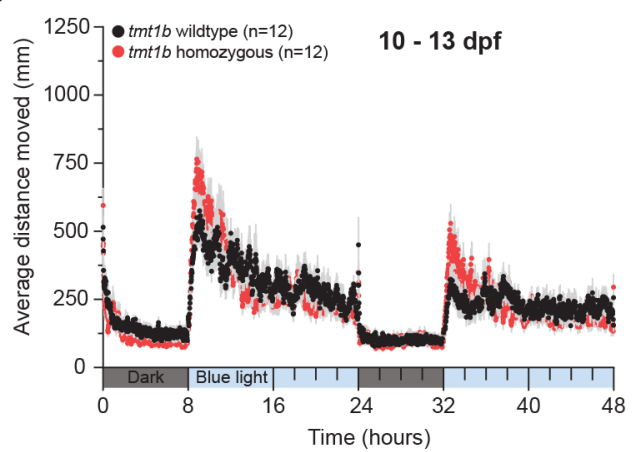

G

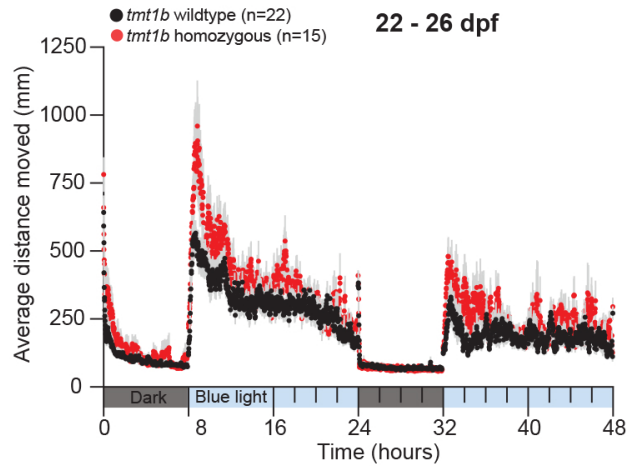

I

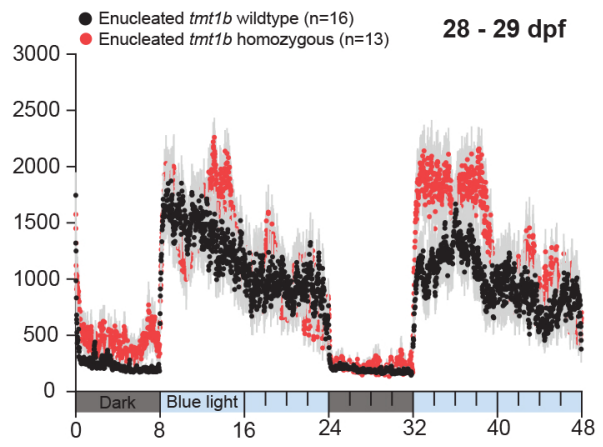

C
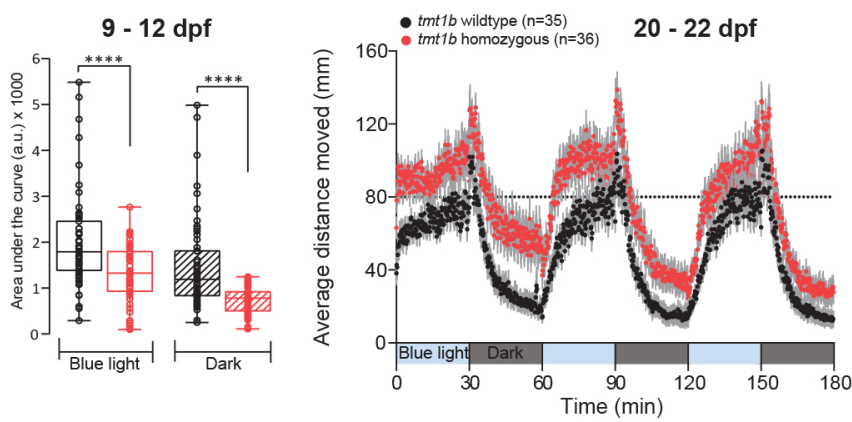

F

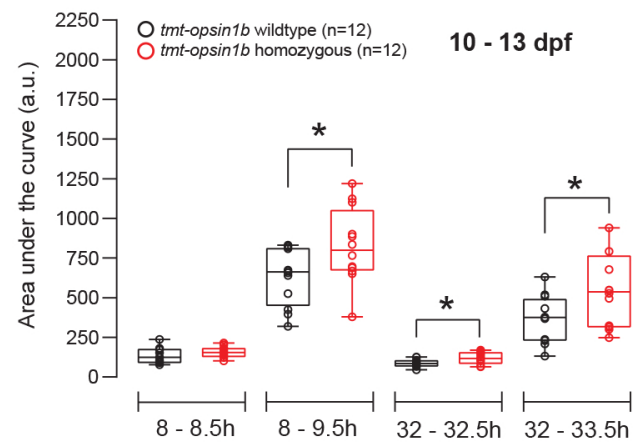

H

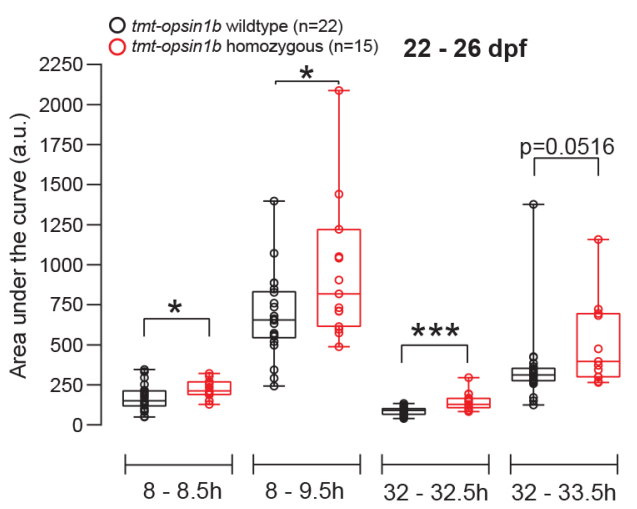

J

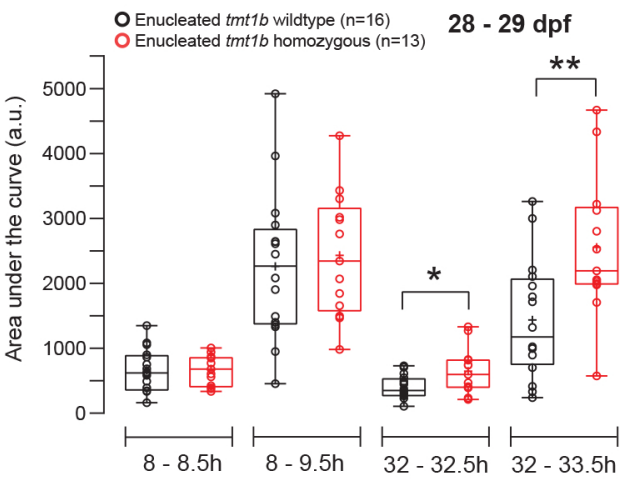

20 - 22 dpf

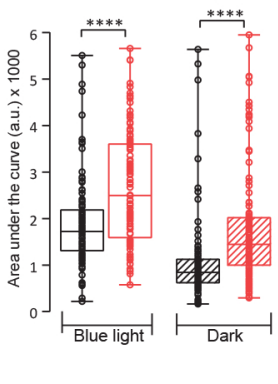


A



B

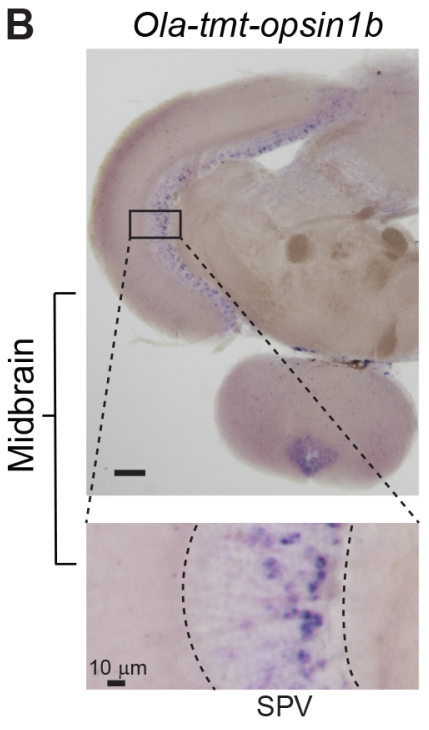

D Ola-tmt-opsin $1 b$

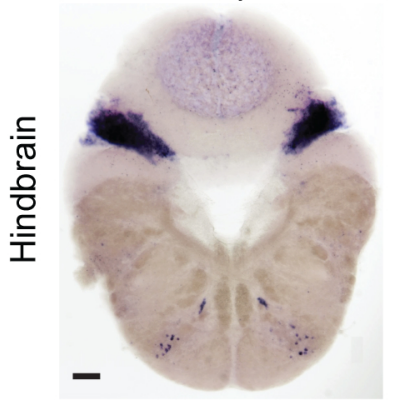

C Ola-tmt-opsin2

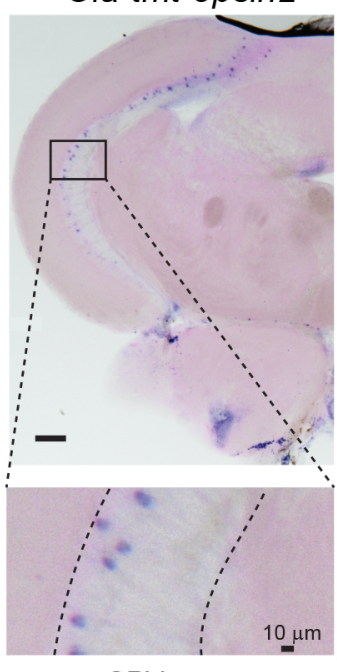

E

$$
\text { SPV }
$$


$\mathbf{F}$

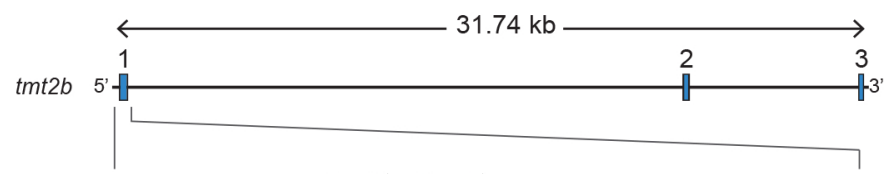

tmt2b exon 1

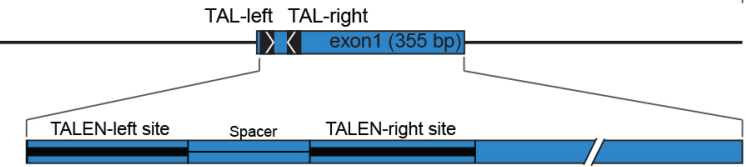

$t m t 2 b^{+/+}$GTTTTCCGGTCAGACtggactcaacttcagctTCAACCAGAGCGATGACCGGGAGC //CAACTCCTGCTTTG tmt $2 b^{-1-}(\Delta-276)$ GTTTTCCGGTCAGACtggctgcagctgcagct-

G
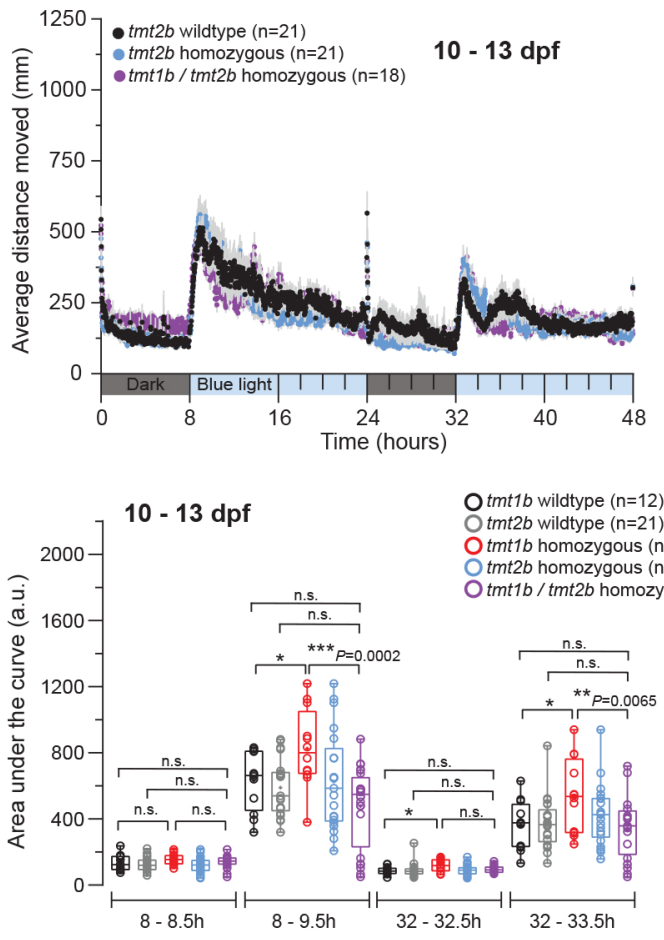

I
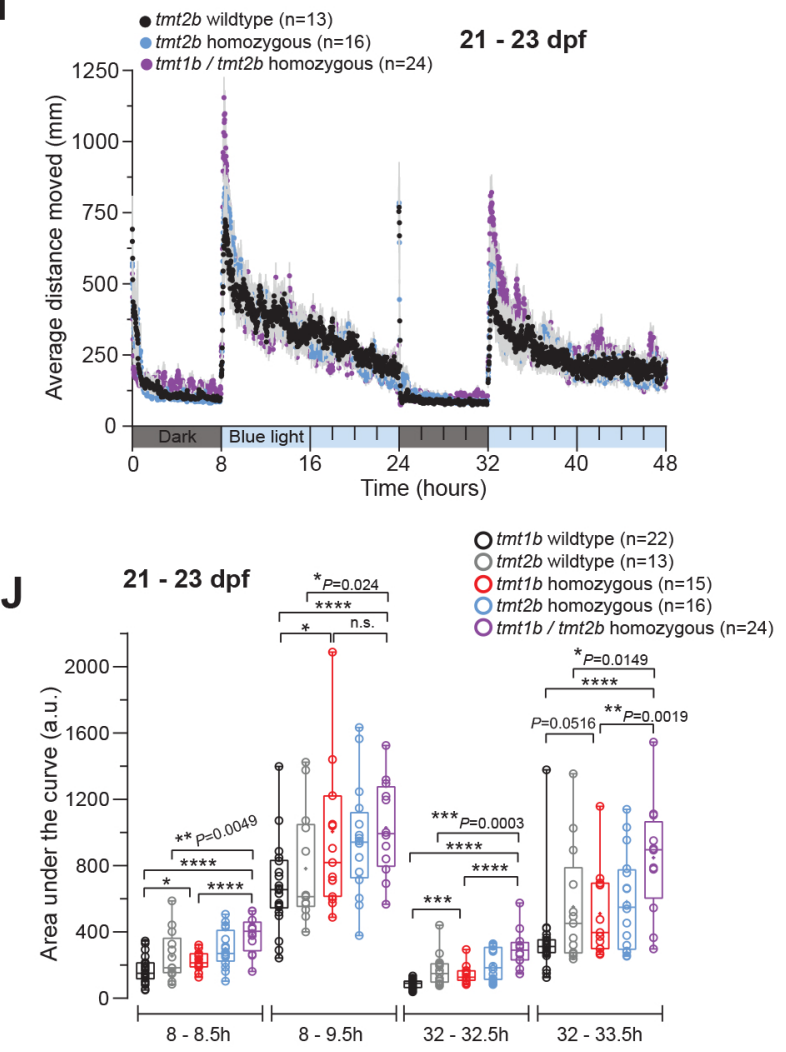
B

A
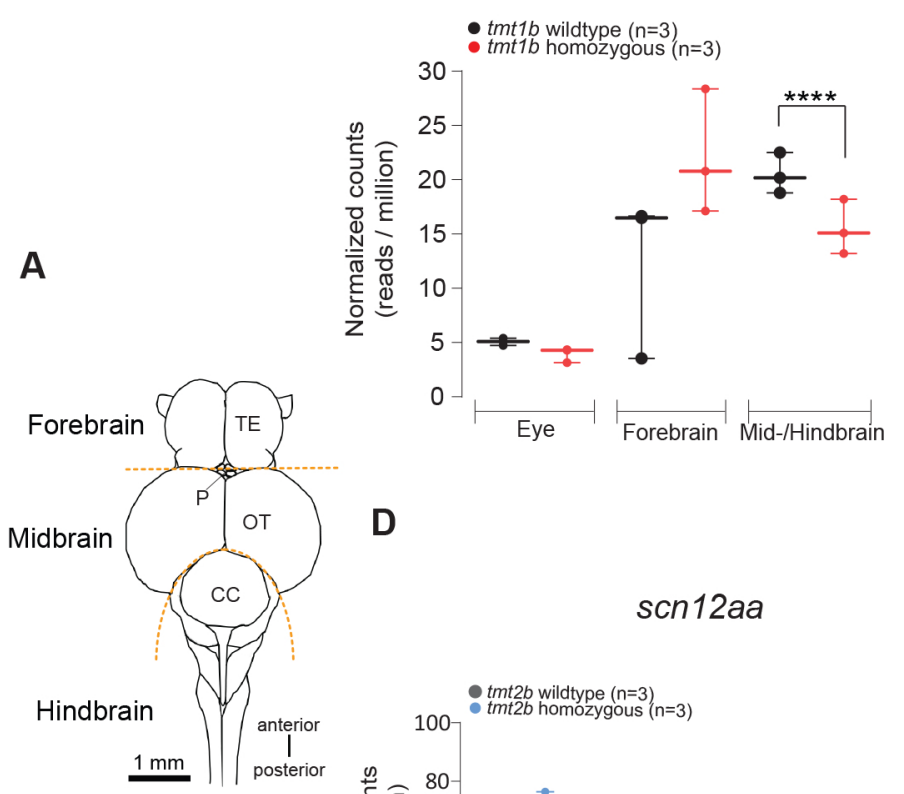

D



C



E




A

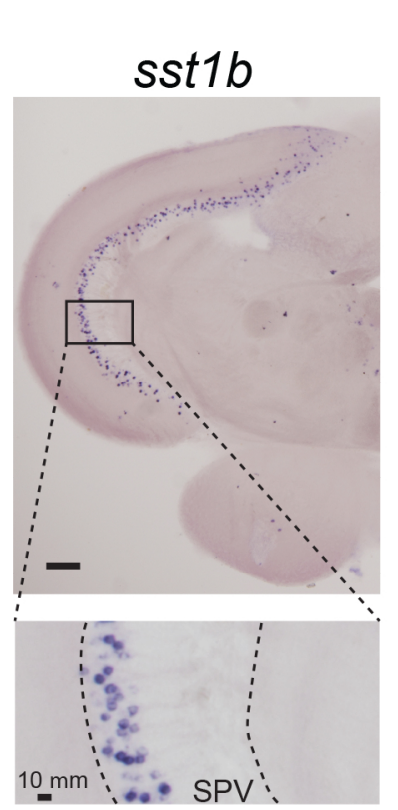

B

\section{sst1b}


sst1b

tmt-opsin $1 b$ wildtype tmt-opsin $1 b$ homozygous


\section{sst1b}

tmt-opsin $1 b$ wildtype tmt-opsin $1 b$ homozygous 
A

\section{Long day (16h light / 8h dark)}

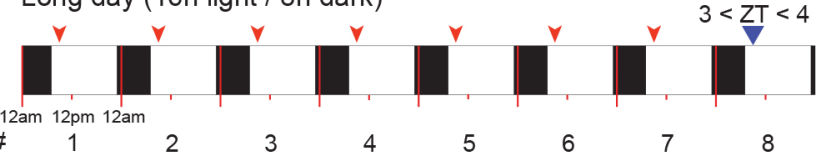

Day \#

Short day (8h light / 16h dark)

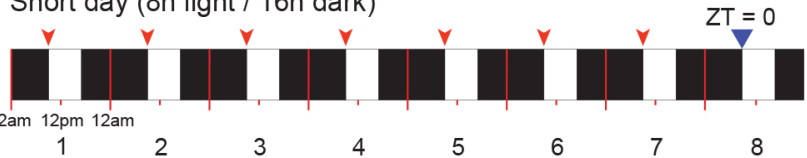

Day \#

1

C sst $1 b$
tmt-opsin $1 b$ wildtype

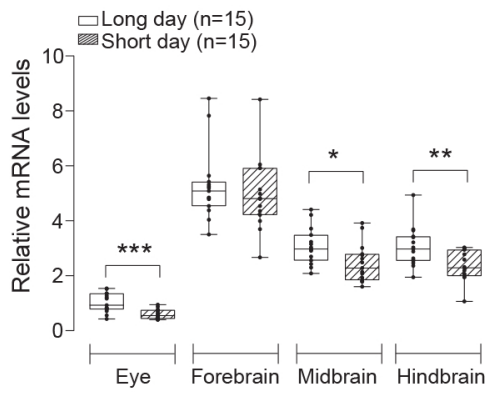

D

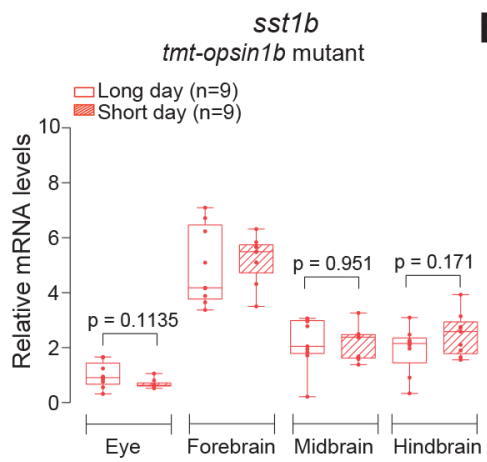

fish room illumination

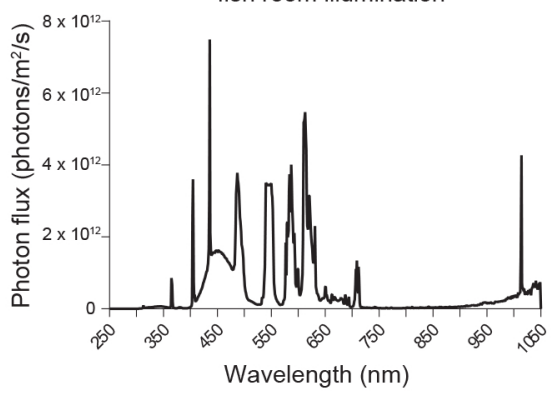

E

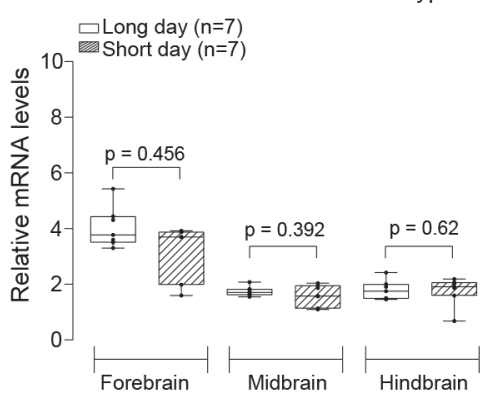




\section{Figure legends}

Figure 1: Mutations in Ola-tmt-opsin1b cause light-dependent differences in an avoidance response test.

(A) Genomic locus of the Ola-tmt-opsin $1 b$ gene and corresponding mutant alterations. TALEN binding sites: black boxes; Exons: grey boxes; Inserted/substituted nucleotides: red; deleted nucleotides: “-”. (B) Confocal images of anti-Ola-TMT-1b (green) and nuclear DAPI (blue) staining in wildtype and tmt-opsin $1 b(\Delta-2 / \Delta-2)$ mutant coronal tectal slices. SPV: stratum periventriculare. Scale bars: $20 \mu \mathrm{m}$. (C) Schematic of the behavioral setup. (D) Behavior size discrimination tuning curve of larvae reacting to moving dots presented in ascending size order. (E) Behavior analysis as in (D), performed in a different laboratory with a different computer screen and surrounding (Light intensity $=2.2 \times 10^{13}$ photons $/ \mathrm{m}^{2} / \mathrm{sec}$ ). (F) Avoidance responses to the dots displayed in a shuffled size manner. $(\mathbf{G})$ Avoidance responses in the ascending dot size paradigm with background light reduced to $35 \%$ of its initial intensity $(35 \%$ light intensity $=0.77$ $\mathrm{x} 10^{13}$ photons $/ \mathrm{m}^{2} / \mathrm{sec}$ ). (H) Total avoidance (area under the curve) under different light intensities. (G) Spectra of the emitted computer screen light used for the $100 \%$ (black) and the 35\% light intensity (orange). Data presentation: (D-G) mean ( \pm s.e.m.); (H) single bar: median, box: first and third quartile, whiskers: min and max; all assays done with 9-12 dpf larvae. a.u.: arbitrary units.

Figure 2: Mutations in Ola-tmt-opsin $1 b$ impact on activity levels upon light changes in a partly age-dependent and partly eye-independent manner

$(\mathbf{A}, \mathbf{C}, \mathbf{E}, \mathbf{G}, \mathbf{I})$ Average distance moved during alternating 30 minutes blue light / dark intervals $(A, C)$ and 8 hours dark / 16 hours blue light periods during two consecutive days (E,G,I) from larvae $(A, E)$, juvenile fish $(C, G)$ and enucleated juvenile fish $(\mathrm{I})$. Each data point represents the mean ( \pm s.e.m.) distance moved for the preceding 10 seconds $(A, C)$ or 1 minute $(E, G, I)$. Colored boxes along the $\mathrm{x}$-axis represent light condition. (B,D) Locomotor activity (measured as area under the curve:AUC) during the entire blue light vs. dark intervals of the trial. $* * * * P \leq 0.0001$. (F, $\mathbf{H}, \mathbf{J})$ Locomotor activity (measured as AUC) during the first 30min and 90min after light is turned ON during the 2 days trial. $(\mathrm{F}) * P_{(8-9.5 \mathrm{~h})}=0.0331 ; * P_{(32-32.5 \mathrm{~h})}=0.0247 ; * P_{(32-33.5 \mathrm{~h})}=0.0323 ;(\mathrm{H}) * P_{(8-}$ $8.5 \mathrm{~h})=0.0330 ; * P_{(8-9.5 \mathrm{~h})}=0.0299 ; * * * P_{(32-32.5 \mathrm{~h})}=0.0002 ;(\mathrm{J}) * P_{(32-32.5 \mathrm{~h})}=0.0192 ; * * P_{(32-33.5 \mathrm{~h})}=$ 0.0056. For all panels, (black) tmt-opsin $1 b$ and (red) tmt-opsinlb homozygous single mutant. Box 
plots: single bar: median, box: first and third quartile, whiskers: min and max; n.s.: no significance; a.u.: arbitrary units.

\section{Figure 3: Individual and combined differential impact of Ola-tmt-opsin $1 b$ and $O l a-t m t-o p \sin 2$} on daytime rest levels

(A) Schematized lateral view of an adult medaka brain. Dashed lines: position of sections in B,C and D,E. NDIL: nucleus diffuses of lobus inferiosis (of hypothalamus). (B,C,D,E) In situ hybridization (ISH) of tmt-opsin1b (B,D) and tmt-opsin2 (C,E) on coronal sections from $\mathrm{Cab}$ wildtype fish. SPV: stratum periventriculare. Scale bars: $100 \mu \mathrm{m}$. (F) Genomic locus of the Olatmt-opsin 2 gene and corresponding mutant. TALEN binding sites: black boxes; Exons: blue boxes; Inserted/substituted nucleotides: red; deleted nucleotides: “-”. (G,I) Average distance moved profile during a 2 consecutive days trial on a 16h:8h blue-light/dark photoperiod of (G) 10-13 dpf larvae and (I) 21-23 dpf juvenile fish. Each data point represent the mean ( \pm s.e.m.) distance moved for the preceding 1 minute. Colored boxes along the x-axis represent light condition. (H,J) Locomotor activity (assessed by area under the curve) during the first 30 minutes or 90 minutes after light is turned $\mathrm{ON}$ along the 2 days trial of $(\mathrm{H})$ larvae and $(\mathrm{J})$ juvenile fish. Comparison between Ola-tmt-opsin $1 b$ wildtype and mutant refers to Fig. $2 \mathrm{~F}(\mathrm{H})$ and Fig $2 \mathrm{H}(\mathrm{J})$. For all panels: $* * * * P<0.0001$. Box plots: single bar: median, box: first and third quartile, whiskers: min and max; n.s.: no significance; a.u.: arbitrary units.

\section{Figure 4: Mutations in Ola-tmt-opsin1b and Ola-tmt-opsin2 cause differential transcript changes in specific brain parts.}

(A) Schematic drawing of the adult medaka brain in dorsal view. Dotted lines indicate the dissection boundaries (TE: telencephalon; P: pineal organ; OT: optic tectum; CC: corpus cerebelli). Note: part of the posterior hypothalamus is dissected with the midbrain, as it is attached to its ventral part and technically unfeasible to reliably separate. (B) Normalized transcript read counts $(* * * *$ adjusted $P$-value $<0.001)$ for sst $1 b$. (C) mRNA transcript levels for sst $1 b$ in brain areas and eyes. Decreased $s s t 1 b$-levels are unique to tmt-opsin $1 b$-/- mutants and are compensated by the tmt-opsin2-/- mutant. (D) Normalized transcript read counts (**** adjusted $P$-value $<$ 0.001) for scn12aa. (E) mRNA transcript levels for scn12aa in brain areas and eyes. Increased levels of $\operatorname{scn} 12 \mathrm{a} a$ are unique to tmt-opsin2-/- mutants, and compensated for when both tmt-opsin $1 b$ 
and tmt-opsin 2 are jointly mutated. Box plots: single bar: median, box: first and third quartile, whiskers: min and max, each displayed data point corresponds to one individual biological replicate. a.u.: arbitrary units.

Figure 5: sst1b levels are reduced in specific cells in the tectum, posterior hypothalamus and hindbrain in tmt-opsin $1 b-/-$ medaka brains.

In situ hybridization for sstlb performed on coronal sections of midbrain (A) and hindbrain (B) from $\mathrm{Cab}$ wildtype fish. Magnification of boxed areas of the optic tectum are displayed below. SPV: stratum periventriculare. Note that sst $1 b$ identify a distinct tectal population of dorsal cells and on the ventral area of the hindbrain (compare with Fig. 2B,D). (C,D) Comparison of sst1b expression in wildtype vs. tmt-opsin $1 b$ mutant sibling coronal brain sections reveals specific reduction (denoted by arrows) in interneurons of the tectum and reticular formation, and in the posterior hypothalamus. Scale bars: $100 \mu \mathrm{m}$.

\section{Figure 6: sst1b is regulated by photoperiod and depends on the eye and tmt-opsin1b}

(A) Schematic representation of the two different photoperiods assigned to 2 distinct cohort of tmtopsin $1 b$ wildtype and tmt-opsin $1 b$ mutant adult fish. Black boxes: dark period; White boxes: white light period. Red arrowheads: feeding time; Blue arrowheads: time when fish were sacrificed and the brain dissections made. (B) Light spectrum of the light used in (A). (C-E) mRNA sst1b transcript expression levels in tmt-opsin $1 b$ wildtype (C), tmt-opsin $1 b$ mutant (D) siblings and in surgically enucleated Cab wildtype adult fish (E). The downregulation of sst $1 b$ transcript levels seen in the normal wildtype group $(* P=0.0184, * * P=0.0088, * * * P=0.0005)$ is absent in the tmt-opsin $1 b$ mutant and eye enucleated fish. Box plots: single bar: median, box: first and third quartile, whiskers: min and max. Each data point represents one biological replicate. 
A

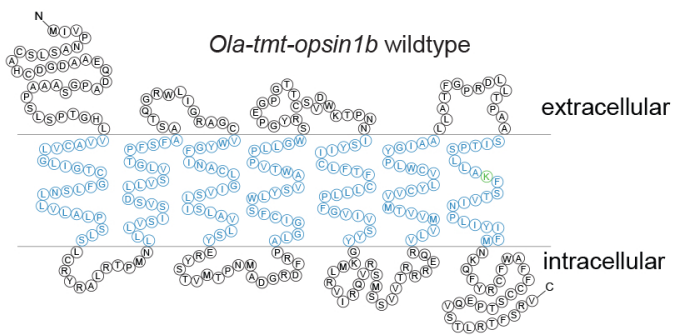

B

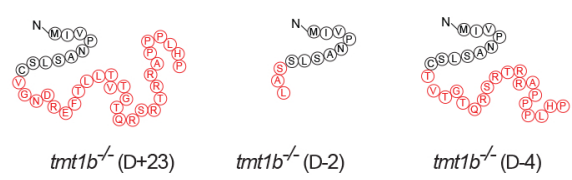

E

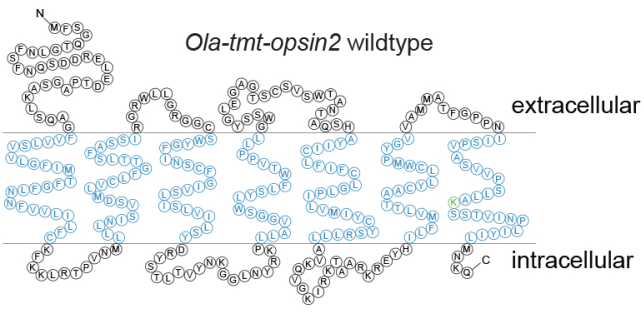

$\mathbf{F}$

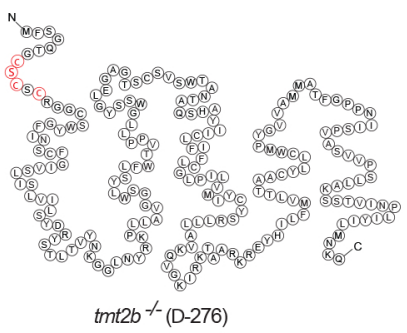

G

GENOTYPE

tmt1b wildtype DNA sequence ATGATCGTTCCCAACGCGAGCCTGAGCTGCGCGCACTGTGACGGGGACGCAGCGGAGC Protein translation MIVPNASLSCAHCDGDAAE

tmt1 $\boldsymbol{b}^{\%}(\mathbf{\Delta + 2 3 )}$ DNA sequence ATGATCGTTCCCAACGCGAGCCTGAGCTGCGTTGGGAACGATCGATTCACGCTGCTGACTGTGACGGGGACGCAGCGGAGC Protein translation MIVPNASLSCVGNDRFTLITFTGTQRSRTRRAPPPLHP*

tmt1 $\%(\Delta-2) \quad$ DNA sequence ATGATCGTTCCCAACGCGAGCCTGAGCTCCGC--ACTGTGACGGGGACGCAGCGGAGC Protein translation MIVPNASLSSAL*

tmt1 1 \% $(\Delta-4)$ DNA sequence ATGATCGTTCCCAACGCGAGCCTGAGCTGC ACTGTGACGGGGACGCAGCGGAGC

Protein translation MIVPNASLSCTVTGTQRSRTSSAPPPLHP*

tmt2b wildtype DNA sequence ATGTTITCCGGTCAGACTGGACTCAACTTCAGCT CAACCAGAGCGATG Protein translation MFSGQTGLNFSFNQSD

tmt $2 b^{-1}(\Delta-276)$ DNA sequence ATGTTTCCGGTCAGACTGGCTGCAGCTGCAGCTProtein translation MFSGQTGCSCS
H

\begin{tabular}{|c|c|c|}
\hline \multicolumn{3}{|c|}{ Panel 1D } \\
\hline & \multicolumn{2}{|c|}{ genotype } \\
\hline allele & wt & homozygous \\
\hline$\Delta+23$ & 14 & 4 \\
\hline$\Delta-2$ & 14 & 16 \\
\hline$\Delta-4$ & 3 & 3 \\
\hline TOTAL & 31 & 21 \\
\hline
\end{tabular}

\begin{tabular}{|lrrr|}
\hline \multicolumn{4}{c}{ Panel 1E $100 \%$ intensity } \\
& \multicolumn{2}{c}{ genotype } \\
allele & wt & \multicolumn{2}{c}{ homozygous } \\
$\Delta+23$ & & 0 & 0 \\
$\Delta-2$ & & 22 & 20 \\
$\Delta-4$ & & 0 & 0 \\
\hline TOTAL & 22 & 20 \\
\hline
\end{tabular}

\begin{tabular}{|c|c|c|}
\hline \multicolumn{3}{|c|}{ Panel 1F } \\
\hline & \multicolumn{2}{|c|}{ genotype } \\
\hline allele & wt & homozygous \\
\hline$\Delta+23$ & 2 & 6 \\
\hline$\Delta-2$ & 3 & 15 \\
\hline$\Delta-4$ & 3 & 6 \\
\hline TOTAL & 8 & 27 \\
\hline
\end{tabular}

\begin{tabular}{|lrrr|}
\hline \multicolumn{4}{|c|}{$\begin{array}{c}\text { Panel } 1 G \text { 35\% intensity } \\
\text { genotype }\end{array}$} \\
allele & wt & \multicolumn{2}{c}{ homozygous } \\
$\Delta+23$ & & 4 & 3 \\
$\Delta-2$ & & 9 & 5 \\
$\Delta-4$ & 0 & 0 \\
$\Delta-2 / \Delta-4$ & 1 & 2 \\
TOTAL & 14 & 10 \\
\hline
\end{tabular}

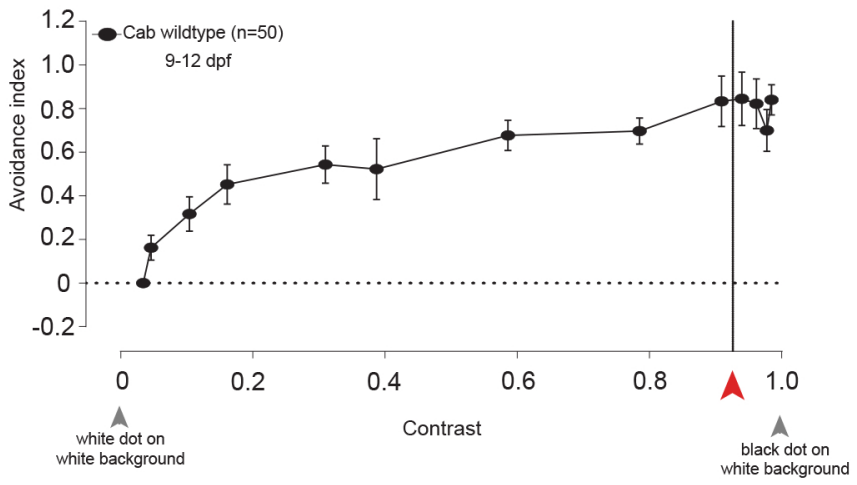


A



C

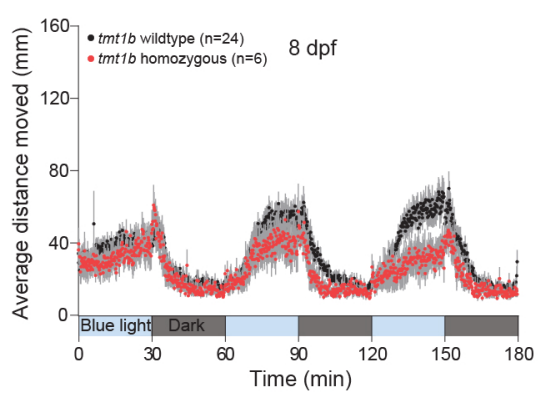

G

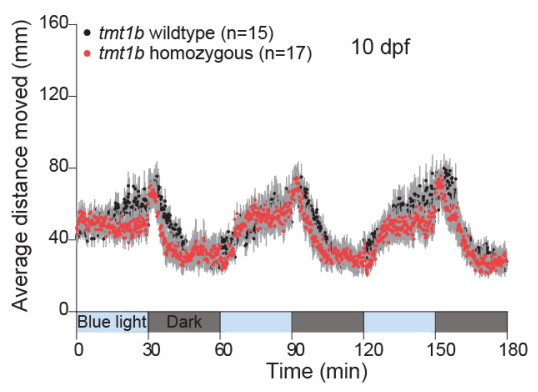

H
D $8 \mathrm{dpf} \quad$ E
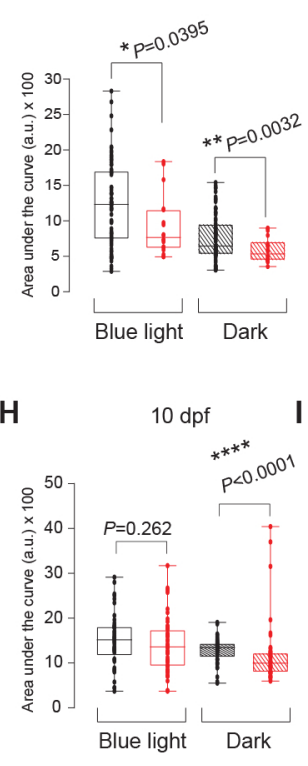

$20-22 \mathrm{dpf}$

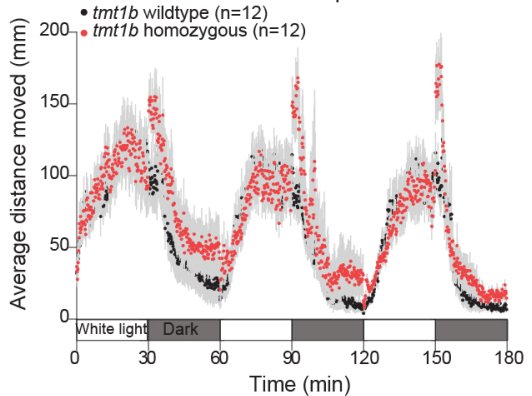

M

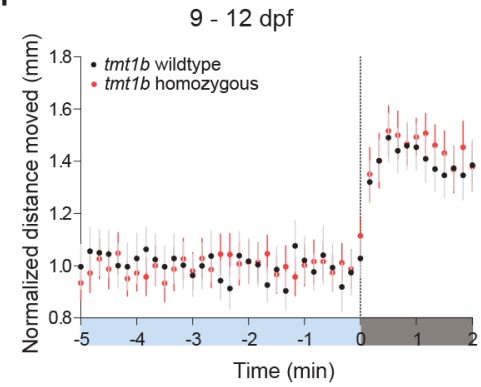

L

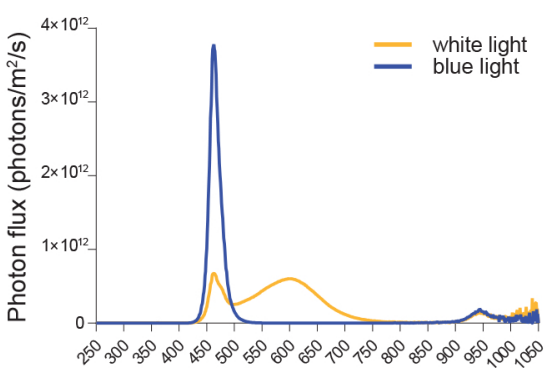

Wavelength $(\mathrm{nm})$

F
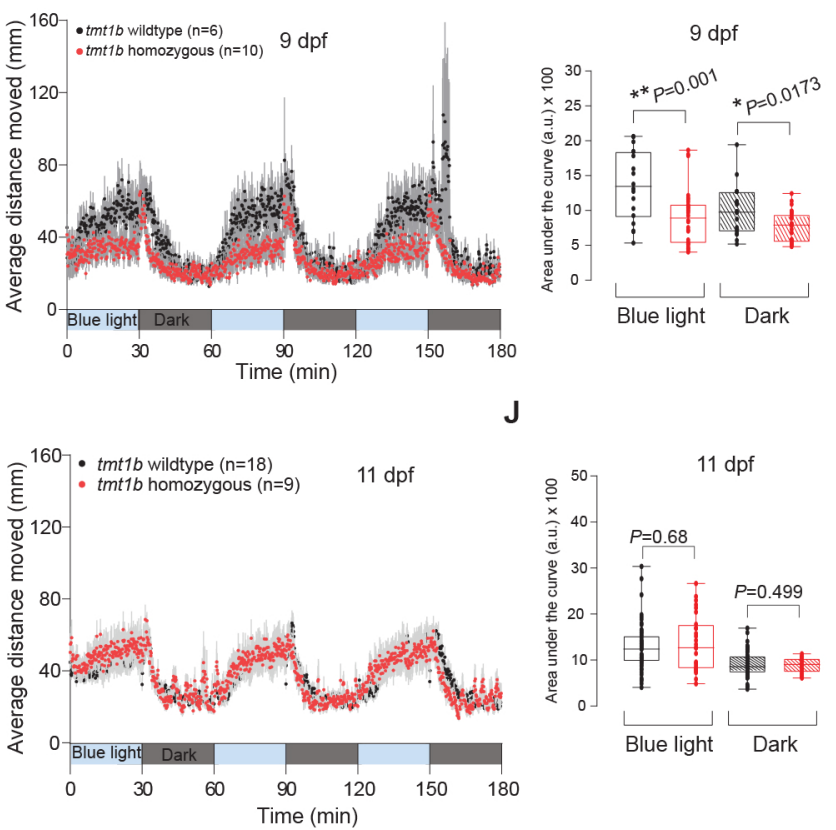

$20-22 d p f$



N

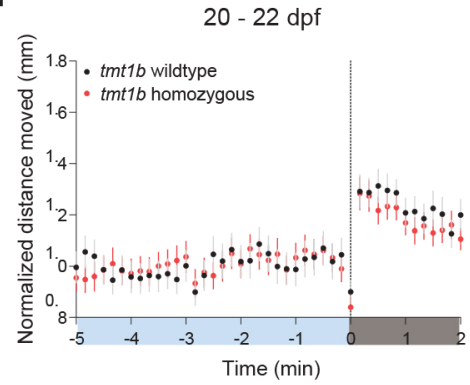




\section{Supplementary Figure and Movie legends}

\section{Supplementary Figure 1: refers to Figure 1}

(A-F) Schematic of the predicted amino acid (aa) sequence for wildtype TMT-Opsin1b (A), wildtype TMT-Opsin2 (E), and corresponding recovered single TMT-Opsin1b (B-D) and TMT-Opsin2 (F) mutants. Blue aa: transmembrane domains; Green aa: Lys296 forming the chromophore's Schiff base; Red aa: substituted amino acids. (G) Partial Olatmt-opsin1b and Ola-tmt-opsin2 genomic DNA sequence and corresponding aa codon translation for wildtype and corresponding mutants that were used interchangeably along the study (grey: TALEN binding sites sequences; blue: recognition enzymatic site for BssHII; red: inserted nucleotides and substituted aa; *: stop codon). All the tmt-opsin1b mutants had in common the disruption of the BssHII recognition site (used for mutagenesis PCR confirmation) and a premature stop codon which led to a possible N-terminal truncated and hence null-functional protein. $(\mathbf{H})$ Quantification of the number and identity of the wildtype and mutant allele's siblings used in each trial. (I) Avoidance index levels of Cab wildtype larvae different contrast luminance levels between the moving dot (constant $20^{\circ}$ size) and the white computer screen background. Red arrowhead indicated the contrast level measured at 35\% light intensity. Note that at this contrast value, the level of avoidance is comparable to the avoidance levels revealed at higher contrasts.

Supplementary Fig. 2: refers to Figure 2

(A) Locomotor activity (measured by area under the curve) from 20-22 dpf juvenile fish across all white light and dark phases (1min duration each) in the photokinesis trial. ****P $<0.0001$. (B) Light spectra of the different LED lights used in the photokinesis assays (white light: orange trace; Blue light: blue trace). (C-L and M-N) Average distance moved profile during alternating 30 minutes light / dark intervals and corresponding locomotor activity (as measured by the area under the curve) for $8 \mathrm{dpf}(\mathrm{C}, \mathrm{D}), 9 \mathrm{dpf}(\mathrm{E}, \mathrm{F}), 10 \mathrm{dpf}(\mathrm{G}, \mathrm{H})$ and $11 \mathrm{dpf}(\mathrm{I}, \mathrm{J})$ larvae, as well as 20-22 $\mathrm{dpf}(\mathrm{K}, \mathrm{L})$ juvenile fish. Each data point represents the mean $( \pm$ s.e.m.) distance moved for the preceding 10 seconds. $(\mathbf{K}, \mathbf{L})$ Dark photokinesis response normalized to the average of the 5 minutes preceding darkness for larvae $(\mathrm{K})$ and juvenile fish (L). All panels: (black) tmt-opsin1b wildtype and (red) tmt-opsin $1 b$ mutant. 
Colored boxes along the $\mathrm{x}$-axis represent condition. Box plots: single bar: median, box: first and third quartile, whiskers: min and max. a.u.: arbitrary units.

\section{Supplementary Video 1: refers to Figure 1E}

Example of a $10 \mathrm{dpf}$ tmt-opsin $1 b$ wildtype larvae performing the avoidance assay during the ascending size dot assay. Large dots elicit a distinct avoidance behavior.

\section{Supplementary Video 2: refers to Figure 1E}

Example of a $10 \mathrm{dpf}$ tmt-opsinlb single homozygous larvae performing the avoidance assay during the ascending size dot assay. Large dots elicit a distinct avoidance behavior. Note that the displayed number of avoidances is higher when compared with wildtype larvae. 